\title{
BAU VON LEHMZIEGEL-PLATTFORMEN IN DER EISENZEIT. DER HORIZONT DER BAUMEISTER IN GEOKTCHIK DEPE UND IZAT KULI (TURKMENISTAN) KURZE NOTIZEN VON DER FELDARBEIT IM GANG
}

\author{
Joaquín M ${ }^{\text {a }}$ Córdoba Zoilo \\ (Universidad Autónoma, Madrid)
}

\begin{abstract}
ZUSAMMENFASSUNG
Einige, allgemein anerkannte, kulturelle Merkmale der Eisenzeit Zentralasiens, werden von der heutigen Forschung in Frage gestellt oder anders angegangen. Unter ihnen befinden sich die damals $g$ übernommen und an die Bräuche dieses Gebietes angepasst worden ebräuchlichen Bautechniken, so wie die möglicherweise von aussen empfangenen Einflüsse. Die spanisch-turkmenische Expedition in Dahistan liefert interessante neue Daten zu dieser Diskussion.
\end{abstract}

SCHLÜSSELWORTE

Zentralasien, Eisenzeit, Lehmziegel-Plattformen, Bautechniken.

\section{RAISING MUD BRICK PLATFORMS. POSSIBLE REFERENCES OF THE MONUMENTAL BUILDINGS OF GEOKTCHIK DEPE AND IZAT KULI (TURKMENISTAN) DURING THE IRON AGE}

\begin{abstract}
Some cultural features of the Iron Age in Central Asia, generally well accepted, are now being revised or focused in a different way by modern research. The construction techniques used and the possible influences received by the new states are counted among the newly analyzed elements. The Turkmen and Spanish mission in Dahistan brings interesting and novel facts to the question
\end{abstract}

\section{KEY WORDS}

Central Asia, Iron Age, mud brick platforms, construction techniques

\section{1.- Einleitung}

In der herkömmlichen Fachliteratur über Zentralasien wird allgemein anerkannt, dass die großen Lehmziegel-Plattformen ein charakteristisches Merkmal der Architektur der Eisenzeit sind (1500/1100-329 v. C.) ${ }^{1}$. Auf ihnen errichtete man die offiziellen Gebäude. So sprach man seinerzeit von "befestigten Zitadellen" die Anfang des ersten Jahrtausend v. C. "die lokalen Herrscher in ihren Mauern beherbergt hätten, getrennt von der gemeinen Bevölkerung, die in kleinen Häusern zu ihren Füßen wohnte". ${ }^{2}$ Man hob damals diese Bauten als etwas einzigartiges, Zentralasien eigenes, hervor, ohne zu beachten, dass man, was Struktur und Funktion betrifft, ja das gleiche von den riesigen Plattformen der großen, assyrischen Hauptstädte wie Kalhu oder Dūr-Šarrukīn, hätte sagen können.

Als wir unsere Arbeiten in Dahistan begannen, schienen mir diese Gesichtspunkte vollkommen logisch. Sie passten gut in die Chronologie und von den überlieferten Texten her, schien eine solche Beziehung zwischen Mesopotamien und Zentralasien durchaus

\footnotetext{
${ }^{1}$ O. Lecomte, H.-P. Francfort, R. Boucharlat, M. Mamedow.- "Recherches archéologiques récentes à Ulug Dépé (Turkmenistan)" Paléorient 28, nr. 2 (2002): 123-132. Tafel 1: 124

${ }^{2}$ V. M. Masson, V. I Sarianidi.- Central Asia. Turkmenia before the Achaemenids. Thames and Hudson Southampton 1972: 162.
} 
vernünftig. Nach unseren ersten Grabungsarbeiten in Geoktchik Tepe und Izat Kuli, scheinen unsere vorläufigen Ergebnisse, sie großteils zu bestätigen. Gleichzeitig bescheren uns diese Arbeiten Informationen, die die Einzigartigkeit der Kulturen dieser Gegend bekräftigen. Trotz der großen Zeitspanne zwischen den Plattformen der Eisenzeit (eingeschlossen denen aus unseren Fundstätten) und, z.B. dem monumentalen Lehmziegelbau aus dem dritten Jahrtausend in Altyn Depe, ${ }^{3}$ weisen unsere Funde auf eine regionale Einzigartigkeit hin. Andrerseits zeigen sie auch Beziehungen zu den Steppengebieten, so wie Ideen und Einflüsse, die aus so entfernten Gegenden wie das Assyrien des ersten Jahrtausends stammen.

\section{2.- Die Lehmziegel-Plattformen während der Eisenzeit in Zentralasien. Wissenschaftlicher Stand und neue Ergebnisse}

Die Lehmziegel-Plattformen oder Zitadellen, waren ein zentrales Element des Stadtbildes der Eisenzeit in diesem Gebiet. Da meine Untersuchung zwei Hauptrichtlinien verfolgt (die Bauten selbst und ihre Funktion) müssen wir hier sowohl die bisherigen Hypothesen, wie die Zweifel, die anhand der neueren Ausgrabungen aufkommen, berücksichtigen. Ich möchte auf keinen Fall Fehler aufzeigen, sondern die bahnbrechenden Ergebnisse der sowjetischen Forschungsarbeiten den heutigen Funden anpassen (Abb. 1). Anhand der sowjetischen Forschungen in so unterschiedlichen Gebieten wie der Misrianebene, (das alte Vehrkānā/ Hirkanien/ Dahistan), dem nördlichen Gebiet zu Füßen des Koppet Dagh (Parthien/ Etek) oder den Tälern der Flüsse Tedyen und Murghab, mit ihren riesigen, über hundert Kilometer breiten Deltas ${ }^{4}$, die das alte Marguš/Margianien befruchteten, betrachtete man die Funktion (Herrscherresidenz) und die Bautechnik (Lehmziegel) der Plattformen, als ein hier allgemeines Merkmal der Eisenzeit. Wenn man vorschlug, dass die befestigten LehmziegelPlattformen die Gebäude der Herrscher beherbergten und dass um sie herum die Wohnviertel der Bevölkerung lagen', spiegelte dies die Ergebnisse mehrerer Ausgrabungen wieder (besonders in Izat Kuli (Dahistan) oder Yaz Depe und Takhirbai (Margianien)) oder der Prospektionen, die man in nahegelegenen Gebieten oder auf regionaler Ebene durchgeführt hatte, um die archäologische Landkarte der Gegend auszuführen, da diese Bauten damals und auch heute, noch sichtbar und topographisch eindrucksvoll, an vielen Stätten aus dieser Epoche stehen. Nach den ersten Arbeiten von M.E. Masson, A.A. Marushenko, B.A. Litvinski un A.A. Roslyakov ${ }^{6}$, war in Dahistan V. Mijailovich Masson der erste, der, dank der Sondierungen und Prospektionen, die von ihm in der Misrianebene durchgeführt wurden, diese Bauten wissenschaftlich beschrieb. In Izat Kuli erwähnte er eine große, sechs Meter hohe Zitadelle?. In Madau Depe nahm man an, dass der höchste topographische Punkt, der $13 \mathrm{~m}$ hoch lag, den Standort und das Ausmaß der, ebenfalls aus Lehmziegeln gebauten, Zitadelle, anzeigte ${ }^{8}$. Damals wurde auch an anderen Orten dieses Gebietes gegraben oder in den nachfolgenden Jahrzehnten, wenigstens teilweise, Sondierungen ausgeführt ${ }^{9}$ und dieses

\footnotetext{
${ }^{3}$ V. M. Masson.- Altyn-Depe. The University Museum, University of Pennsylvania, Philadelphia 1988: 55-80.

${ }^{4}$ M. Julivert.- Desiertos. Clases, distribución y ocupación humana. Publicacions de la Universitat de València, València 2015: 116-119.

${ }^{5}$ V. M. Masson, V. I. Sarianidi.- Op. cit. (1972): 161-162.

${ }^{6} \mathrm{Ph}$. Kohl.- Central Asia Palaeolithic Beginnings to the Iron Age / L'Asie central des origines à l'Âge du Fer. Éditions Recherche sur las Civilisations, Paris 1984: 203.

${ }^{7}$ V. M. Masson.- "Pamjatniki kultury arkhaicheskogo Dakhistana v jugo-zapadnoj Turkmenii”, TRYDY JUTAKE VII (1956): 385-458. Zu Izat Kuli: 390-402. Siehe 390.

${ }^{8}$ V. M. Masson.- Op. cit.: 402-422. Vid. 403.

${ }^{9}$ Z. B. Chialik oder Chiglik Depe, nicht weit von Tangsikildzha, deren $6 \mathrm{~m}$ hohe Zitadelle von einem Gebäude mit neun Räumen gekrönt war. Es ist interessant, dass Gutliev feststellte, dass die $3 \mathrm{~m}$ hohe Festungsmauer, die die Zitadelle auf allen Seiten umgab, nicht aus Lehmziegeln errichtet war, sondern nur aus Lehmschlag oder Paksha. So G. Gutliev.- "Raskopki na kholme Chialik (Chiglik-Depe)” KD IV (1972): 47-55.
} 


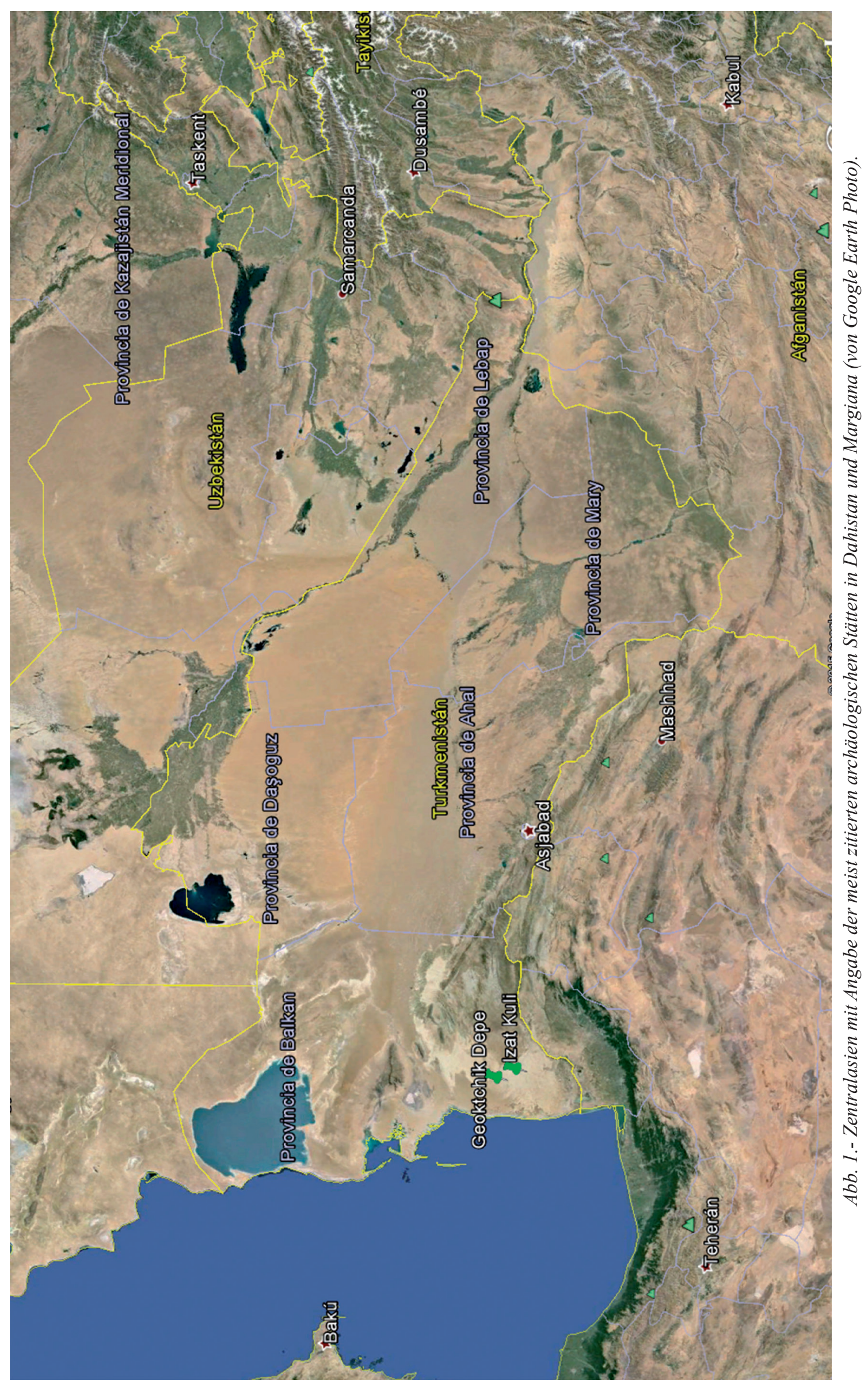


typologische Schema der wichtigsten Siedlungen der Eisenzeit (die hier archaische DahistanKultur genannt wurde) würde lange unverändert bleiben.

Wenn wir von einer möglichen Plattform in Elken Depe, in dem nördlichen Gebiet zu Füßen des Koppet Dagh, nicht weit von Aschgabat, absehen, die augenscheinlich nicht ausgegraben worden ist $\mathrm{t}^{10}$, waren die Beispiele in Margianien ausschlaggebend für die typologische Definition dieser Bauten, die ich hier untersuche, vor allem die Plattformen in den Siedlungen Yaz Depe und Takhirbai. Die erste (Abb. 2 und 3), die einzige, die wirklich ausgegraben worden ist, bildete eine $12 \mathrm{~m}$ hohe Zitadelle. Von diesen zwölf Metern, waren acht eine Plattform aus harten Lehmziegeln. Auf dem südlichen Ende dieser Plattform, stand ein 30 x 34m großes Gebäude, mit äusserst länglichen, meist gangartigen Räumen und dicken Lehmziegelmauern. Dieses Gebäude besaß mit Sicherheit einen zweiten Stock. Neben ihm lag ein 26 x 7m großer, offener Raum, den V. M. Masson als Eingang eines Palastes oder Tempels deutete ${ }^{11}$.

Die andere wichtige, mögliche Lehmziegel- Plattform wurde in Takhirbai entdeckt. Sie wurde durch eine rechteckige Erhöhung angedeutet, die in der Mitte des archäologischen Areals lag, und die mit ihrer auffälligen Form und ihren 7 m Höhe und 100m Seitenlänge, auf ein Bauwerk dieser Art wies ${ }^{12}$.

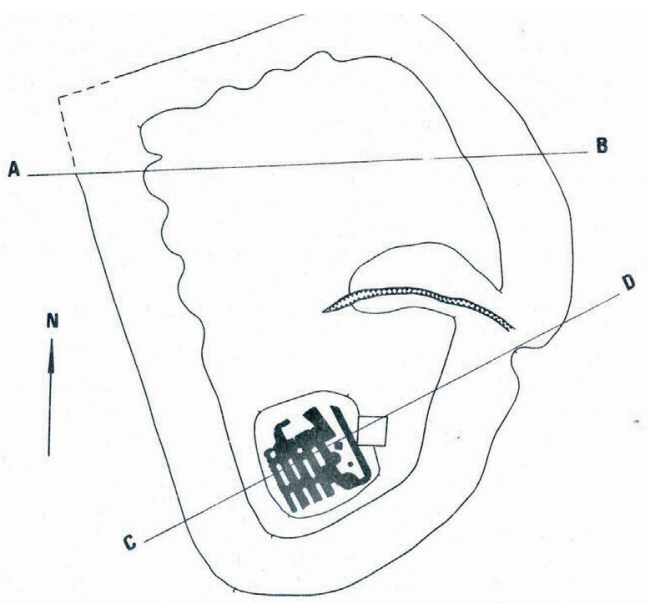

Abb. 2.- Yaz Depe-Plattform mit Angabe der Abschnitte in Abb. 3 (nach V. M. Masson, V.I. Sarianidi, 1972: 162, Abb. 26, a).

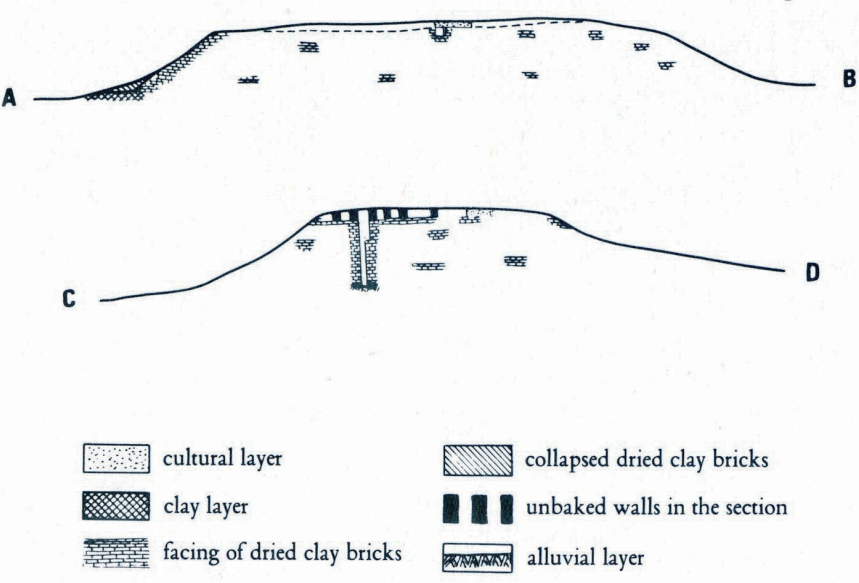

Abb. 3.- Schnitte durch die Yaz depe-Plattform (nach V. M. Masson, V. I. Sarianidi, 1972: 163, Abb. 26, b).

Zusammenfassend kann man sagen, dass nach der Information, die man aus den Grabungen und Prospektionen gewonnen hatte, die Annahme, dass die wichtigsten Siedlungen der Eisenzeit durch eine große Lehmziegel-Plattform gekennzeichnet waren, wohlbegründet war. Wenngleich aber die Funktion dieser Bauten auch nicht in Frage gestellt wird (man ist sich einig, dass diese Plattformen ein Unterbau für die öffentlichen Gebäude waren, ohne Näheres über die Art dieser Gebäude zu sagen) kommen hinsichtlich der Bautechniken Fragen auf, deren Antwort unser Verständnis der Fähigkeiten der alten Baumeister und der wirtschaftlichen Ressourcen dieser Gesellschaften, erweitern würde.

\footnotetext{
${ }^{10}$ A. A. Marushchenko.- "El'ken Depe (Otchët o raskopkakh 1953, 1954 I 1956 gg.)”, Trudy Instituta Istorii Arkheologii I Etnografii Akademii Nauk Kirgizskoy SSR V (1959): 54-109.

${ }^{11}$ V. M. Masson.- Drevnezemledel cheskaka cultura Margianii, Nauka, Moscú 1959. Siehe vor allem 68-82.

${ }^{12}$ V. M. Masson.- Op. cit. (1959): 63-65.
} 
In Geoktchik Depe, zum Beispiel, in der heutigen Misrianebene, im Gebiet des alten Dahistan, stellte O. Lecomte fest, dass das, was ursprünglich als $11 \mathrm{~m}$ hoher Hügel und Überrest einer Siedlung des archaischen Dahistan verstanden worden war, in Wirklichkeit eine riesiges Bauwerk darstellte, das aus zwei großen Plattformen mit einer unerwarteten Funktion bestand. Die erste zeigte in ihrer Mitte einen äusserst großen, fast viereckigen Raum, in dessen nördlicher Wand sich fünf Räume öffneten und der 16,60 m lang, 15,20 $\mathrm{m}$ breit und 13,40 m hoch war. Seine Umgebungsmauern sind verschieden dick, mit einem Maximum von $13 \mathrm{~m}$ und aus den, für die archaische Dahistan-Kultur typischen, harten Lehmziegeln erbaut. An der südlichen Seite der ersten Plattform, hatte man etwas später eine zweite angebaut, auf der man oben mehrere Räume fand ${ }^{13}$. Die stratigraphischen Schnitte im Norden, Osten und Westen, schienen Anhäufungen von Lehmziegeln zu bestätigen. Aber in der obersten Schicht des östlichen Schnittes, stellte man eine grosse Ansammlung von Lehmschlag/Paksha fest und weiter unten, auf dem heutigen Hang, fand man Lehmziegelschichten bis zu einer Tiefe von $20 \mathrm{~m}^{14}$, wo man den Rand des Gebäudes vermutete. Im Norden schien diese Lehmansammlung $13 \mathrm{~m}$ stark. Was ich aber hier hervorheben möchte ist, dass schon damals, trotz der architektonischen und technischen Festigkeit der Mauern des Hauptraums und der Oberflächen, die stratigraphischen Schnitte im Osten und Westen Anzeichen dafür zeigten, dass die Masse des Gebäudes nicht aus eigentlichen Lehmziegeln bestand. Es stimmt, dass der monumentale Bau in Geoktchik Depe keine Plattform im Sinne V. M. Massons ist, dennoch wirft er uns in unserer ersten Grabung Fragen auf, die wir später untersuchen werden (Abb. 4 und 5).

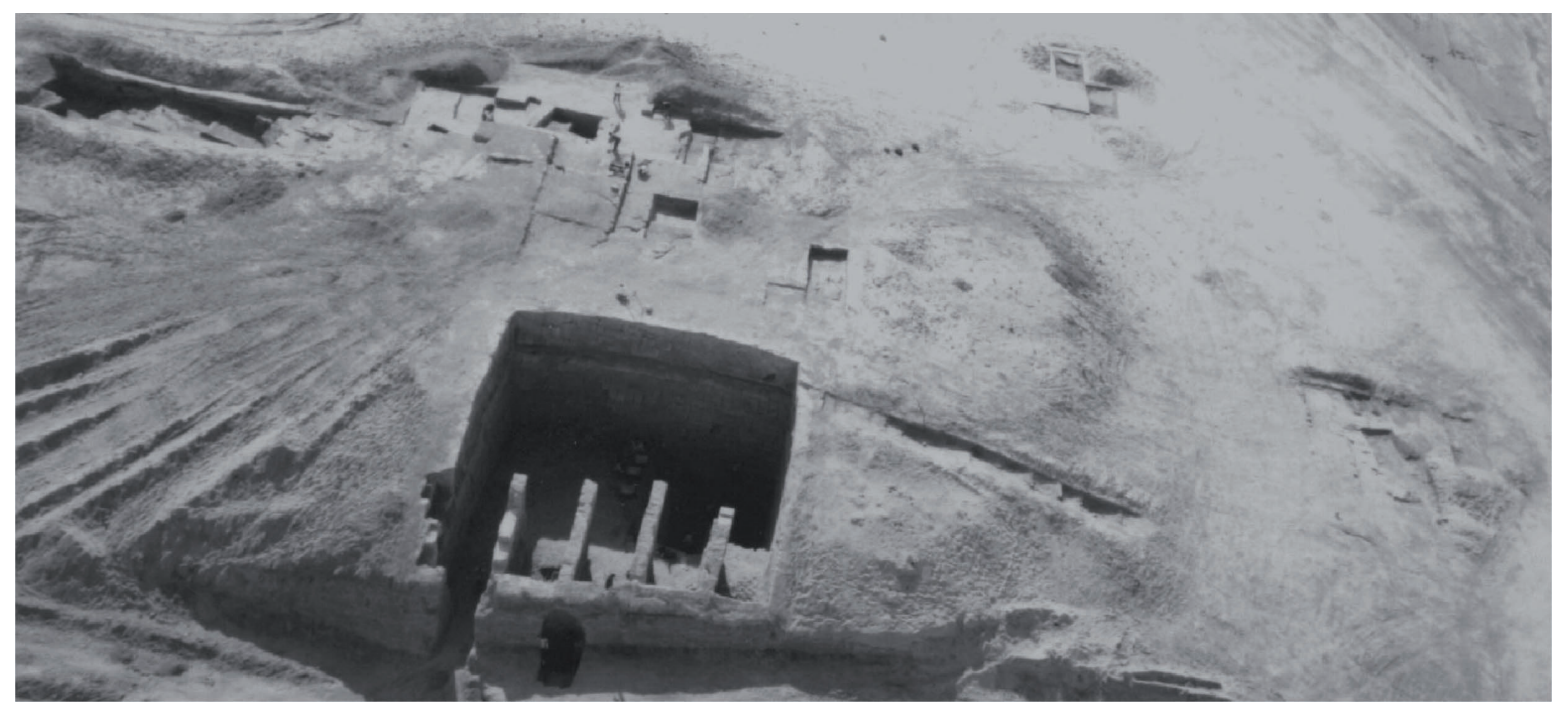

Abb. 4.- Luftaufnahme von Geoktchik depe in Richtung S, aufgenommen während der archäologischen Ausgrabungen der französischen und turkmenischen Mission (durch großzügige Abtretung von O. Lecomte).

\footnotetext{
${ }^{13}$ O. Lecomte.- "Vehrkānā y Dehistan: late farming-communities of south-west Turkmenistan from the Iron Age to the Islamic period", Parthica 1 (1999): 135-170.

${ }^{14}$ O. Lecomte.- Op. cit. (1999): 153.
} 


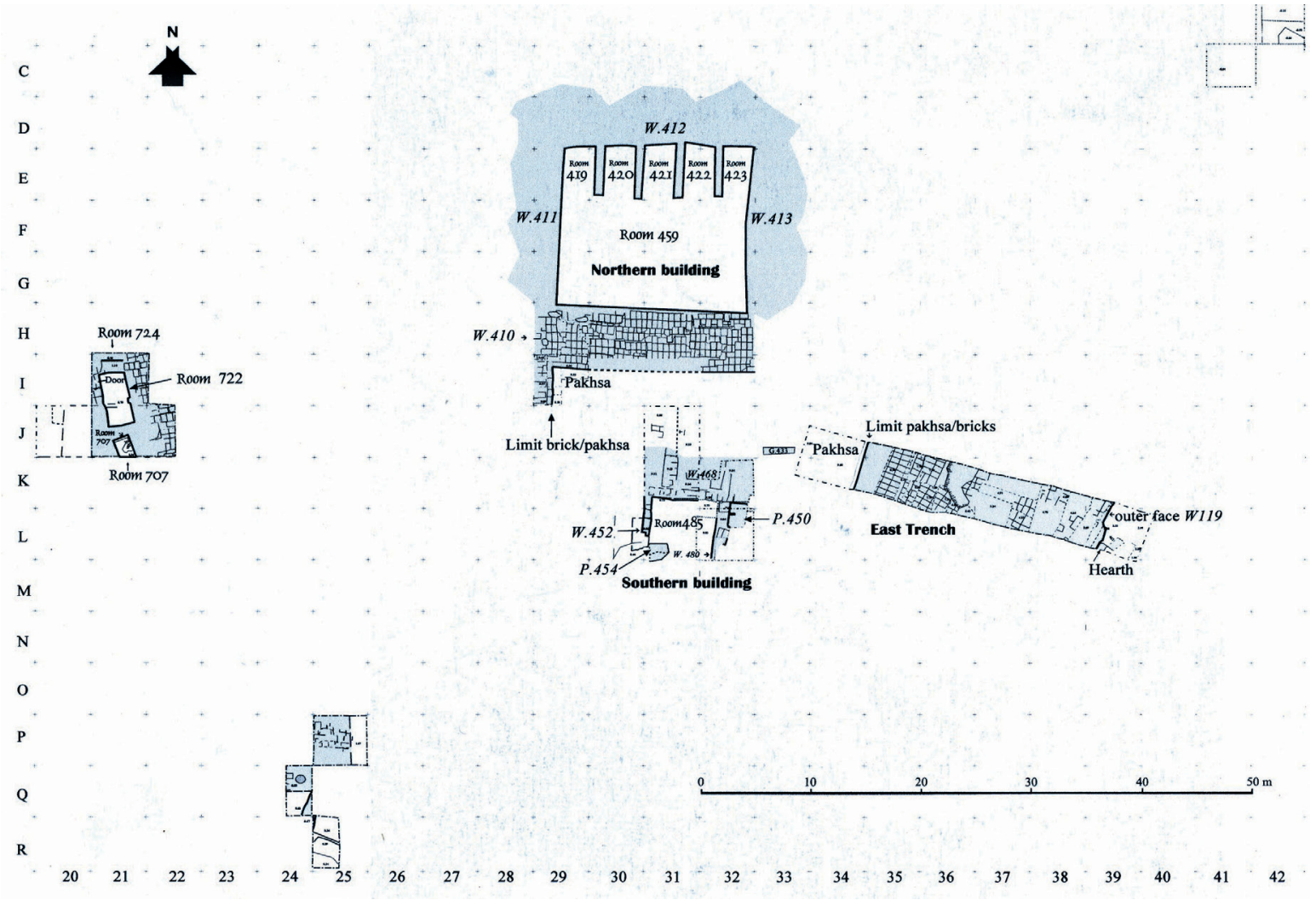

Abb. 5.- Geoktchik Ausgrabungsplan (nach O. Lecomte, 1999: 153, Abb. 5).

Andrerseits hat eine italo-turkmenische Expedition in Takhirbai das Bausystem der Plattform dort, die nach Masson ganz aus Lehmziegeln bestand, neu definiert. Dank eines tiefen stratigraphischen Schnittes von ihrer Höhe bis zu ihrem Fuß, zeigte M. Cattani, dass diese Plattform in Wirklichkeit aus Lehmschlag/Paksha gebaut worden war, der außen mit Lehmziegeln verkleidet war ${ }^{15}$. Wenngleich die Interpretation der Funktion dieser Plattformen klar scheint (obwohl man eine Diskussion ihres Kontexts und ihrer möglichen Vorbilder vermisst), waren diese zwei Ergebnisse, die Fragen zur effektiven Praxis der alten Baumeister in diesem Gebiet aufwerfen, Anlass für mich, die handwerklichen Fertigkeiten und die Techniken der Baumeister der Eisenzeit näher zu untersuchen. Wenn wir in Anbetracht nehmen, dass der monumentale Bau von Altyn Depe tausend Jahre lang keine Kontinuität fand, und nirgends nachgebildet wurde (wenigstens, nach unserem heutigen Wissensstand), müssen wir untersuchen, ob während der Eisenzeit, dieser Epoche, die neue Staaten und Königshäuser hervorbrachte, es vielleicht äussere Einflüsse waren, die das Vorbild für diese visuelle Bestätigung der Macht, die das Stadtbild prägte, lieferten. Diese großen Plattformen, scheinen ja ganz klar eine gewisse politische Absicht auszudrücken.

\section{3.- Herkömmliche Gebräuche der Baumeister in Mesopotamien und der assyrischen Yazira}

Das Gebiet der alten Kulturen des nahen und mittleren Ostens ist sehr gross und besitzt je nach Ort, unterschiedliche Ressourcen. Seit Anfang der menschlichen Geschichte, haben

\footnotetext{
${ }^{15}$ M. Cattani.- "Excavations at Takhirbaj tepe (THR-1) (1992-1993). Preliminary notes”, in A. Gubaev, G. A. Koshelenko, M. Tossi (dir.).- The Archaeological Map of the Murghab delta. Preliminary Reports 1990-95. Istituto Italiano per l'Africa e l'Oriente, Roma 1998: 97-104.
} 
großteils die geographischen Gegebenheiten der unmittelbaren Umgebung bestimmt, welche Baumaterialien benutzt wurden ${ }^{16}$. Da in Nordsyrien und der syrischen Levante, in Palästina oder Anatolien, Stein und Holz leicht zu finden waren, wurde beides großzügig von den Baumeistern der Dörfer und Städte eingesetzt. Aber nicht ausschließlich, denn obwohl sie ein Steinfundament besaßen, wurden die Wände oft aus Lehmschlag oder Lehmziegeln errichtet, manchmal mit einem Fachwerk aus $\mathrm{Holz}^{17}$. Selbst dort waren, genau wie es in den Gegenden, die keinen Stein oder Holz zum bauen hatten geschah, die von den Baumeistern und ihren Kunden bevorzugten Baumaterialien, der Lehmschlag, die Lehmziegel und die gebrannten Ziegel ${ }^{18}$, also die "Architektur aus Erde", die das Wesen selbst der Bauten im nahen Orient zu allen Zeiten ausmachte ${ }^{19}$. Im Laufe der Jahrhunderte und auch je nach den finanziellen Möglichkeiten des Auftraggebers, des Wesens der Bauten, der angesammelten Erfahrung, so wie den Innovationen, die allmählich im Bauwesen erfolgten, veränderten sich die Zusammenstellung und die Verhältnisse der Materialien zueinander. Es wurden auch manchmal andere Materialien benutzt, sei es aus rituellen Gründen (z.B. Sand bei Fundamenten und Füllwerken) aber vor allem aus technischen, und so benutzte man oft Schotter, Bitumen, Kalk, horizontale Balken und Balkenroste, aufgeschichtete Matten aus Palmblättern, Seile und Steinquader oder Werksteine. ${ }^{20}$ Der Baumeister (der itinnu ${ }^{21}$ ) ist eine zentrale Figur in der Geschichte der Bautechnik. Zwar begegnen uns in Mesopotamien schon in Tell es-Sawan, in der Samarra Zeit ${ }^{22}$, Andeutungen von der Tätigkeit von Baumeistern, jedoch zeigt sich dieser Beruf in der allgemeinen Geschichte nur selten, wie zum Beispiel in Dokumenten wie dem Codex Hammurabi ${ }^{23}$.

Seit seinen Anfängen, hatte sich der Beruf des Bauarbeiters zu einer immer größeren Komplexität entwickelt, was zur Spezialisierung einiger Arbeiter geführt hatte. Mindestens seit der Obeid Zeit (ich würde sagen, sogar etwas früher), folgten die Bauten einem vorher entworfenem Plan. Damals verfügten ausserdem die Baumeister schon über eine "erstaunliche Kenntnis der Proportionen"24, denn man kannte schon "die Eigenschaften der rechteckigen Dreiecke 3:4:5 und 5:12:13, die erlauben mit Hilfe eines einfachen, mit Knoten versehenen Seils, auf dem Boden rechte Winkel zu zeichnen" "25. Sehr viel später, zur Zeit des assyrischen Kaiserreiches, zählten die "Maurermeister", die die Mauern von Dūr Šarrukīn errichteten, mit der Hilfe von Lehrlingen, die ihnen die Materialien brachten ${ }^{26}$. Diese Rangordnung (Meister, Geselle, Lehrling) war im Laufe der Jahrhunderte entstanden. Aber jetzt wollen wir nur einen

\footnotetext{
${ }^{16}$ O. Aurenche.- Dictionnaire illustré multilingüe de l'architecture du Proche Orient ancient. Maison de l'Orient, Lyon 1977.

${ }^{17}$ R. Naumann.- Architektur Kleinasiens von ihren Anfängen bis zum Ende der hethitischen Zeit. Verlag Ernst Wasmuth, Tübingen 1971.

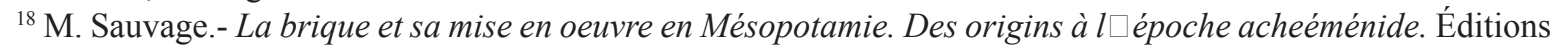
Recherche sur les Civilisations, Paris 1998.

${ }_{19}^{19}$ J. Dethier-- Architectures de terre. Éditions du Centre Pompidou, Paris 1986.

${ }^{20}$ S. A. A. Kubba.- Mesopotamian Architecture and Town Planning from the Mesolithic to the end of the Protohistoric Period c. 10.000-3.500 B.C. BAR International Series 367, Oxford 1987: 159-183.

${ }^{21}$ E. Ebelling,. "Baumeister", RLA 1 (1981): 438-439.

${ }^{22}$ D. G. Youkhana.- Tell es-Sawwan. The Architecture of the Sixth Millennium B.C.. NABU Publicactions, London 1997: 63-64.

${ }^{23}$ J. Sanmartín.- Códigos legales de tradición babilónica. Editorial Trotta, S. A., Madrid 1999. Unter anderen, mehr oder weniger schweren, Tatbeständen, fällt "Artikel” 229 auf, wo gesagt wird, dass falls ein neugebautes Haus einstürzen sollte, weil der Baumeister es nicht ordentlich gebaut hätte und dabei der Hausherr erschlagen würde, der Baumeister hingerichtet werden sollte.

${ }^{24}$ S. A. A. Kubba.- Op. cit. (1987): 132.

${ }^{25}$ M. Sauvage.- Op. cit. (1998): 75.

${ }^{26}$ M. Sauvage.- Op. cit. (1998): 76.
} 
Blick auf die, von den alten Meistern bei dem Bau der großen Plattformen angewendeten Techniken, werfen. Unter "Plattform" verstehen wir hier nicht die so berühmte Ziqurratu, sondern ausschließlich diejenigen, auf denen repräsentative Gebäude errichtet wurden, seien diese religiös, weltlich oder beides zugleich.

Das älteste Beispiel der bei dem Bau von Plattformen angewandten Technik, haben wir im Eridu der Obeid Zeit ${ }^{27}$, da die Daten aus den Grabungsberichten nicht so zahlreich sind, wie man erwarten sollte. Was die Autoren temple-platform oder acropolis-platform nennen, besaß eine dicke, aus Stein gebaute Schutzmauer, die einen dichten Verputz aus Lehmmörtel zeigte ${ }^{28}$. Die Plattform, die von dieser Schutzmauer umgeben war, bestand aus der stratigraphischen Überlagerung älterer Lehmschlag- und Lehmziegelmauern, mit Zwischenschichten und Füllungen aus Sand und Schotter. Am Schluss hatte man alles geebnet, um darauf die neuen Gebäude zu errichten.

Etwa tausend Jahre später, im Dschebel Aruda der Zeit des Ersten Städtebaus, war die Plattform, auf der sich die Tempel erhoben, $50 \mathrm{~cm}$ hoch, aussen von Lehmziegelmauern umgeben und bestand aus unregelmäßigen Steinblöcken und Mörtel ${ }^{29}$. Die viel grössere Terrasse aus derselben Epoche, auf der die Tempel in Habuba Kabira standen, konnte leider nicht ausgegraben werden ${ }^{30}$. Zusammenfassend können wir hier sehen, dass schon diese ältesten Beispiele einer Plattform, eine Wiederverwertung von Überresten älterer Bauten und die Verwendung billiger Materialien, wie Schutt, Sand, Lehm und Schotter zeigen, aber auch, dass sie eine Kenntnis der strukturellen Eigenschaften dieser Materialien beweisen, die es erlaubte, sie richtig zu kombinieren, um die bestmöglichste Stabilität der Bauten zu erreichen.

Im Laufe der Jahrhunderte, und vor allem im Altertum, haben die Baumeister meistens bei ihrer Arbeit die überlieferten Gebräuche und ihre Vorzüge geachtet. Wir wollen hier kein ausgiebiges Inventar der im dritten und zweiten Jahrtausend v. C. gebauten Plattformen aufstellen. Bei den meisten derjenigen, die ausgegraben worden sind, hat man die Bautechnik des Ganzen nicht aufgezeichnet, da man annahm, dass sie aus einer riesigen Masse von Lehmziegeln bestanden, weil ihre sichtbaren Verkleidungen aus Lehm-oder Branntziegeln waren. Wir können aber für unsere Grabungstätten von der Annahme ausgehen, dass, im Falle, dass die Baumeister der vor-achämenidischen Staaten der Eisenzeit ${ }^{31}$ ein, wenn auch vielleicht entferntes, Vorbild hatten, dieses das der nächsten Großmacht sein musste, und das hieße hier Assyrien und vor allem die riesigen Plattformen in Kalhü ${ }^{32}$ oder Dūr Šarrukīn. In Kalhu bezeugen die Grabungen M.L. Mallowans, dass die Plattform, die als Basis für die Monumentalbauten Assurbanipals II. /Aššur-nāșir-apli II. (883-859 v. C.) gedient hatte, ein wirklich kaiserliches Bauwerk war, das Erbe einer langen Bautradition (Abb. 6). Anscheinend (denn die Daten sind leider nicht vollständig), bestand sie aus einer $9 \mathrm{~m}$ hohen

\footnotetext{
${ }^{27}$ F. Safar, M. A. Mustafa, S. Lloyd.- Eridu. Ministry of Culture and Information, Baghdad 1981: 54-59.

${ }^{28}$ F. Safar, M. A. Mustafa, S. Lloyd.- Op. cit. (1981): 57.

${ }^{29}$ C. van Driel, C. van Driel-Murray.- "Jebel Aruda, the 1982 season of excavation, interim report", Akkadica 33 (1983) 1-26. Siehe. 3.

${ }^{30}$ E. Strommenger.- Habuba Kabira. Eine Stadt vor 5000 Jahren. Verlag Philipp von Zabern, Mainz am Rhein 1980: 44.

${ }^{31}$ J. L. Blesa Cuenca.- Los arios. Historia y modos de vida de los pueblos centroasiáticos de la Edad del Hierro. Universidad Autónoma de Madrid, Madrid 2018 (unveröffentlichte Doktorarbeit)

${ }^{32}$ Wir haben hierfür keinen Beweis, da unter den 47.074 Männern und Frauen aus allen Teilen des Kaiserreiches und vor allem unter den 5000 Würdenträgern, die von den angegebenen Völkern geschickt wurden, niemand erwähnt wird, der auf eine Beziehung mit dem Gebiet, das wir untersuchen, hinweisen könnte. Es scheint jedoch nicht unmöglich, dass die Nachricht von dem beeindruckenden Ereignis der Einweihung der neuen Hauptstadt Aššur-nāşir-aplis II. (883-859 v. C.) die letzten Winkel des Reiches erreichte, sogar jene “entfernten Meder” (A. K. Grayson. Assyrian Royal Inscriptions, I. Otto Harrassowitz, Wiesbaden 1976: 172-176. Vid. 176).
} 
Lehmziegelschicht, während ihre tiefsten Schichten aus Sand und Kieseln waren. Auf diesem dichten Hauptteil, lag eine $2 \mathrm{~m}$ dicke Schicht aus Kieselkonglomerat und darauf, noch 2 bis 4m Lehmziegelschichten auf die die Ziegelböden der Gebäude und Höfe der königlichen Zitadelle gelegt worden waren ${ }^{33}$. Ein anderes Vorbild könnte die von Sargon II. / Šarrukīn (721-705 v. C.) in Dūr Šarrukīn errichtete Hauptplattform gewesen sein. Doch wenn man die Angaben von G. Loud liest, wird einem von diesem kompliziertem Bauwerk nur die Mächtigkeit seiner Verkleidung aus Steinquadern klar, die an den Wänden der Plattform sichtbar $w^{3}{ }^{34}$, was vielleicht darauf hinweist, das sie nicht so fest gebaut war, wie die Plattform, die Tiglatfileser I./ Tukultī-apil-Esara (1115-1077 v. C.) errichten lies, um darauf seinen Anu-Adad Tempel zu bauen, und die fünfzig Lehmziegelschichten zählte ${ }^{35}$.

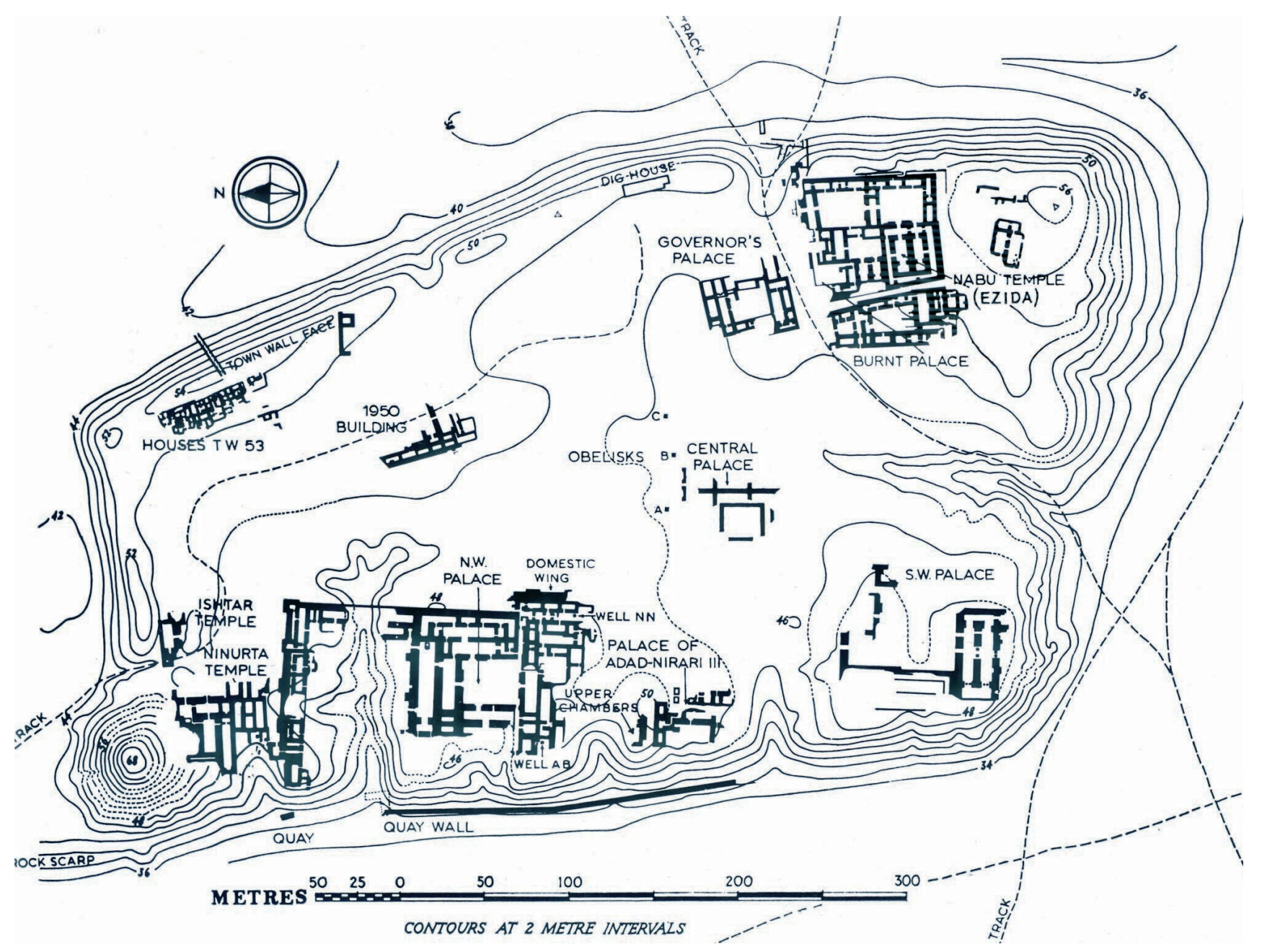

Abb. 6.- Plattform von Kalhu / Nimrud (nach M.E.L. Mallowan 1966: 32, Abb. 1).

Von der Chronologie her und in Anbetracht der bekannten, sowohl kriegerischen wie friedlichen Beziehungen zwischen Assyrern und Völkern des iranischen Ostens und noch weiter entfernt gelegener Gebiete, seien es die berühmten "entfernten Meder" die Asarhaddon/

\footnotetext{
${ }^{33}$ M. E. L. Mallowan.- Nimrud and its Remains. Collins, London 1966: 75-83.

${ }^{34}$ G. Loud, Ch. B. Altman.- Khorsabad, Part I. The Citadel and the Town. The University of Chicago Press, Chicago 1938: 18.

${ }_{35} \mathrm{~S}$. Lackenbacher.- Le roi bâtisseur. Les récits de construction assyriens des origines a Teglatphalasar III. Éditions Recherche sur les Civilisations, Paris 1982:103.
} 
Aššuraha-idin (680-669 v. C.) erwähnt oder andere, die vielleicht auch in den assyrische Texten vorkommen, die wir aber noch nicht identifizieren können, hatten die Menschen Zentralasiens genügend Kenntniss von den großen, mesopotamischen Reichen und pflegten auch genügend Kontakte zu ihnen. Schon vor Asarhaddon unterschieden die Assyrer klar diejenigen Meder, die mit ihnen benachbart waren, von jenen anderen, von denen sie sagten, dass sie jenseits der Salzwüste ${ }^{36}$ oder sogar auf dem "Lapislazuliberg" wohnten.

Zusammenfassend können wir sagen, dass, wenn wir annehmen, dass die Herrscher der Völker des Dahistan der Eisenzeit (1500/1100-500 v. C.) oder die Margianiens, z. B. aus Yaz, ein technisches Vorbild für ihre Plattformen hatten (schliesslich zählten beide zu den "entfernten Medern"), dann wäre dieses Vorbild zum Teil die tausendjährigen Ruinen der alten Plattformen des Hügellandes am Fuße der Berge gewesen, aber, vor allem, die riesigen Plattformen Assyriens, und nicht so sehr Bauten wie der Sommerpalast Nebukadnezars II. / Nabû-kudurrī-ușur (604-562 v. C.) in Babylon ${ }^{37}$ oder die riesigen Plattformen des Darius II. (521-486 v. C.) in Persepolis ${ }^{38}$ oder Susa ${ }^{39}$, deren Datierung später ist als die, die wir für die Bauten der Völker Vehrkānās voraussetzten. Wenngleich Bauten wie die assyrischen, wegen ihrer Größe und den nötigen Baumaterialien ausserhalb ihrer Möglichkeiten lagen, hatten für die Herrscher in Vehrkānā oder Marguš ihre bescheideneren Plattformen dennoch dieselbe Funktion: Sie waren der Sitz der königlichen Macht und der damit verbundenen Symbole. Und was Materialien und Arbeitstechniken angeht, war für sie Altyn Depe, obwohl es einer längst vergangenen Zeit angehörte, das regionale Vorbild für die, von den Baumeistern Zentralasiens überlieferten, Anwendung der Lehmziegel und des Lehmschlages in der Architektur.

\section{4.- Die Techniken der Baumeister Vehrkānās: Neue Ergebnisse}

Die Gebiete Zentralasiens haben tausendjährige Traditionen, genau wie Mesopotamien. Die einzige Ausnahme wären die Einwohner Vehrkānās, eine Gegend, die anscheinend erst Ende des zweiten Jahrtausends bevölkert wurde. Jenseits der anfänglichen Diskussion über die iranische Abstammung dieser Kultur, zeigen uns die Geschichte und die Archäologie, dass ihr Ursprung im Tale des Flusses Sumbar und in der dort entwickelten, bronzezeitlichen Kultur liegt, wie schon vor Zeiten I. N. Khlopin ${ }^{40}$ richtig vorgeschlagen hatte. Wie jede andere eisenzeitliche Kultur Zentralasiens, ist Dahistan ein Produkt der Verschmelzung von Elementen alter, lokaler Traditionen mit den neuen der Andronovo-Kultur ${ }^{41}$, und allem was diese mit sich brachte ${ }^{42}$, so wie mit Einflüssen aus den althergebrachten Beziehungen zu den Gebieten im Südwesten, im Süden und im Südosten. Meines Erachtens, findet all dies seinen Ausdruck in den Bauten und Bautechniken die die Baumeister der Misrianebene in der Provinz Balkan (Turkmenistan), wo wir arbeiten, anwandten (Abb. 7).

\footnotetext{
${ }^{36}$ F. Vallat.- "Expéditions orientales des rois assyriens", Dossiers Histoire et Archéologie 122 (1987): 60-62. Siehe 60.

${ }^{37}$ Besonders R. Koldewey, F. Wetzel.- Die Königsburgen von Babylon. Zweiter Teil. Die Hauptburg und der Sommerpalast Nebukadnezars im Hügel Babil. J. C. Hinrichs'sche Buchhandlung, Leipzig 1932: 41-43.

${ }^{38}$ E. F. Schmidt.- Persepolis I. Strutures. Reliefs. Inscriptions. The University of Chicago Press, Chicago 1953 : 61-63.

${ }^{39}$ D. Ladiray.- "Les données archéologiques", en J. Perrot (dir.).- Le palais de Darius à Suse. Une résidence royale sur la route de Persépolis à Babylone. Presses de l'université Paris-Sorbonne, Paris 2002: 160-223.

${ }^{40}$ I. N. Khlopin.- "Poselenie epokhi bronzi Parkhai-depe" Kratkie Soobshcheniya 142 (1975: 116-121. Vid. 121.

${ }^{41}$ E. E. Kuzmina.- The Origin of the Indo-Arians. E. J. Brill, Leiden, Boston 2007: 455-458.

${ }^{42}$ J. L. Blesa.- Op. cit. (2018): 321.325.
} 


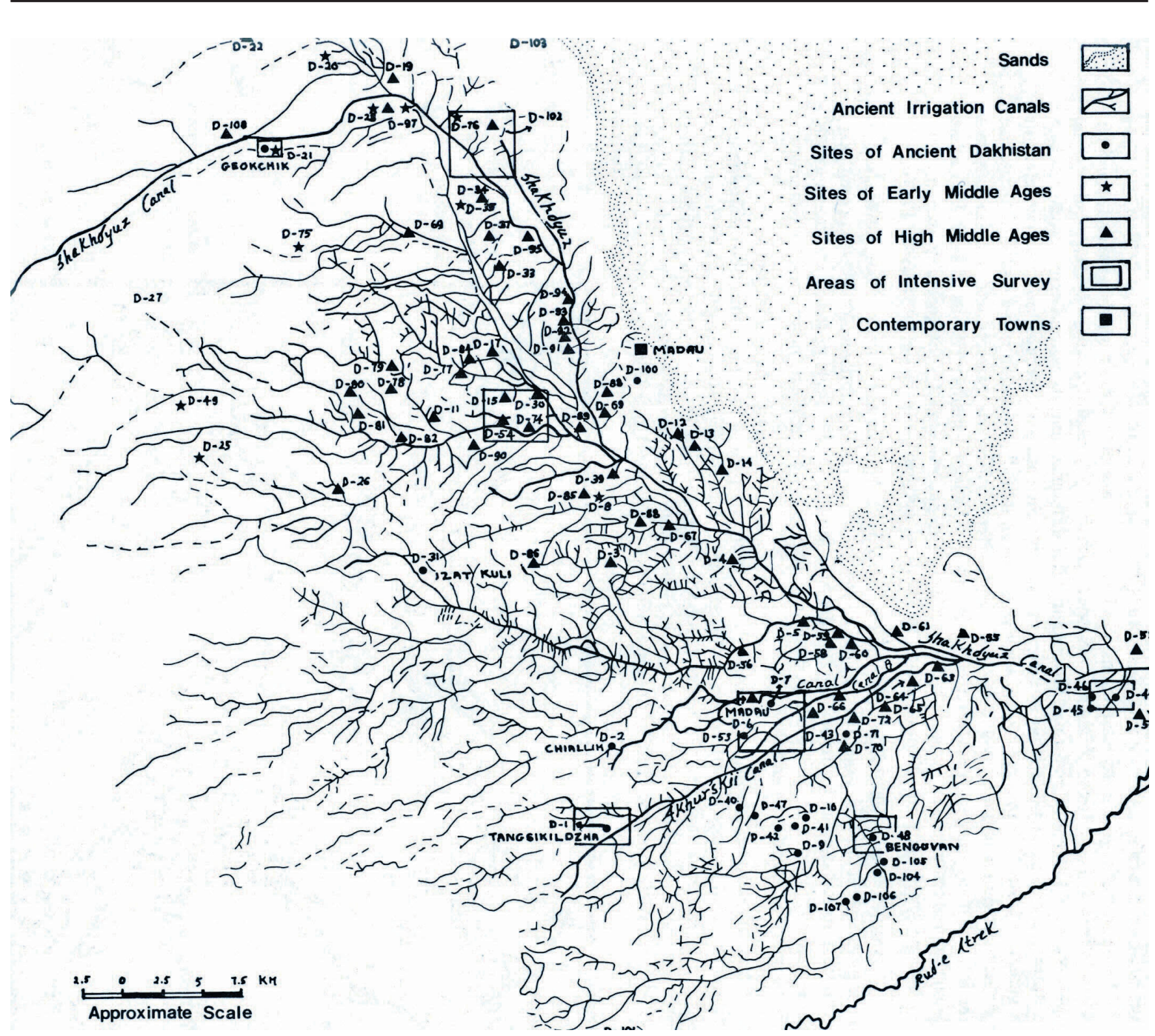

Abb. 7.- Kanal-Netzwerk und Siedlungen in Dahistan. Die Nummern D21 und D31 entsprechen Geoktchik bzw. Izat Kuli (nach Ph. Kohl 1984: 201, Karte 23, hergestellt aus Listisyna 1978).

Die Grabungstätten von Geoktchik und Izat Kuli (von denen wir schon in verschiedenen Publikationen berichtet haben ${ }^{43}$ ), haben eine lange Siedlungsgeschichte, die in den selben Zeitabschnitt fällt. Die erste ist von O. Lecomte in eine Zeit zwischen 1.100 v.C. und 500 v. C. datiert worden ${ }^{44}$, vielleicht etwas früher oder etwas später. Die zweite, die zur archaischen Dahistan-Kultur gezählt wird ${ }^{45}$, deckt zweifellos die drei Perioden dieser Kultur, die durch die Keramik in Izat Kuli selbst ${ }^{46}$ bestimmt werden und innerhalb eines chronologischen

\footnotetext{
${ }^{43} \mathrm{~J} . \mathrm{M}^{\mathrm{a}}$ Córdoba.- «De re rustica» in extrema pars mundi. Agricultura, parcelas y canales en Dehistán durante la Edad del Hierro (1500-500 a. C.). Notas en Izat Kuli", Isimu 18-19 (2015-2016): 391-406. J. Ma Córdoba."La cultura de la Edad del Hierro en Dehistan (1300-500 a. C.). Nuevos trabajos en Geoktchik Depe e Izat Kuli (2014-2015), Informes y trabajos 14 (2016), 188-207. J. Ma Córdoba, M. Mamedov.- "L $\square$ âge du fer au Dehistan. Nouvelles recherches archéologiques turkmènes et espagnoles sur les sites de Geoktchik Depe et Izat Kuli (Province de Balkan, Turkmenistan)", in O. Kaelin, H.-P. Mathys (eds.).- Proceedings of the 9th International Congress on the Archaeology of the Ancient Naer East, Harrassowitz Verlag, Wiesbaden 2016: 601-614

${ }^{44}$ O. Lecomte.- Op. cit. (1999): 156.

${ }^{45}$ E. Atagarriev, G. N. Lisitsyna.- "Raboty nad sostablenien arkheologicheskoy karty Mesehd-Misrianskoy ravninychatskogo masiva”, Karamkumskie Drevnosti III (1970): 166-183. Siehe 181.

${ }^{46}$ E. Muradova.- Poseleniya arkhaicheskovo Dakhistana. Akademiya Nauk Turmenskoi CCP, Ashgabat 1991: 51-71. Siehe Tafel 37: 107.
} 
Bogens liegen, der als ältestes Datum 1280 +/- 50 v. C. hat (siehe Beguvan (LE 1042)) und als jüngstes, das in Tangsilkildzha ${ }^{47}$ aufgenommene, 590 +/- 50 v. C. (LE 1051). Wenn wir bedenken, dass die Eroberung der Staaten Zentralasiens durch die Ächemeniden um $540 \mathrm{v}$. C. begann, passt alles gut zusammen.
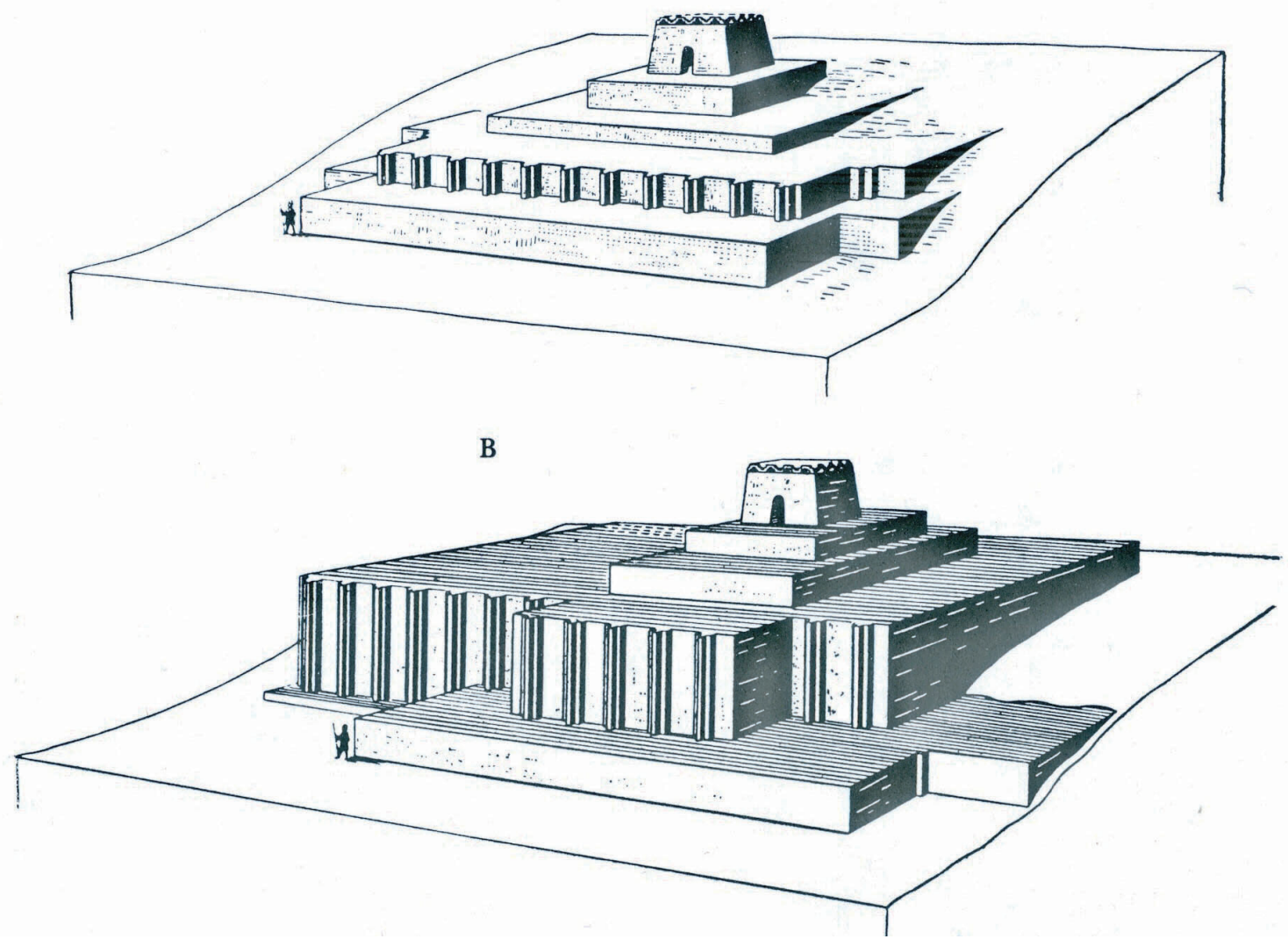

Abb. 8.- Monumentalstrukturen von Altyn depe (nach V. M. Masson 1988: 59, Abb. 18).

Wie wir auf unserer Grabung feststellen können, waren die Baumeister jener Zeit fähig, zwei riesige Bauten aus Lehmziegeln und Lehmschlag oder Paksha zu errichten, der seit tausenden von Jahren ein grundlegendes Element der Architektur Zentralasiens ist ${ }^{48}$. Wir haben zwar Parallelen aus der selben Zeit in Yaz und Takhirbai, aber keine unmittelbaren Vorläufer in Dahistan selbst, das ja, wie wir gesehen haben, erst Ende des zweiten Jahrtausends bevölkert wurde, und auch nicht in anderen Gebieten Zentralasiens, jedenfalls bis jetzt nicht. So müssten wir mögliche technische Vorbilder weit zurück in der Zeit (und weit weg im Raum, wenngleich die vermutlichen Parallelen in Yaz und Takhirbai noch weiter entfernt liegen) in Altyn Depe (Abb. 8), am nördliche Fuße des Koppet Dagh und im dritten Jahrtausend, suchen ${ }^{49}$. Dort errichtete man, auf den Überresten früherer Siedlungen, einen monumentalen Baukomplex aus Lehmziegeln. Diese Überreste gebrauchte man als Füllung für die $20 \mathrm{~m}$ lange und $2 \mathrm{~m}$ hohe Plattform, die als Unterbau der Gebäude diente. Vor dieser Plattform, war der Boden mit einem

\footnotetext{
${ }^{47}$ Ph. Kohl.- Op. cit. (1984): 226.

${ }^{48}$ G. A. Pougatchenkova.- "L'argile matériau fondamental de l'architecture bactrienne", Dossiers d'Archéologie 211 (1996): 8-21. M. Gelin.- "L'argile comme matériau de construction: le pahsa (pisé) et la brique crue", Dossiers d'Archéologie 211 (1996): 22-23.

${ }^{49}$ V. M. Masson.- Op. cit. (1988)
} 
$80 \mathrm{~cm}$ dicken Belag aus Paksha gepflastert, der den Sockel beschützte. Auf der Plattform errichtete man eine Terrasse aus Lehmziegeln, deren Fassade kreuzförmige Pilaster mit drei Vorsprüngen zeigte. Die Oberfläche dieser Plattform hatte man geebnet, so dass sie einen Fußboden formte. In seiner Mitte erhob sich eine Plinthe, auf der eine zweite, etwas kleinere errichtet worden war. Auf dieser stand ein Gebäude ${ }^{50}$, das von kleinen Ausmaßen aber einzigartiger Bedeutung war. Man muss hervorheben, dass, trotz der Wiederverwertung älteren Materials, die neugebauten Teile der Plattform und der Terrasse aus gutem, massivem Lehmziegelverband waren, der sich dem Hang des künstlichen Hügels, auf den sich das Gebäude stützte, anpasste. ${ }^{51}$ Anhand dieser Tatsache und allem, was wir in Geoktchik und Izat Kuli finden, glaube ich, dass mehrere der Techniken, die wir jetzt dort sehen, auf traditionelle, schon damals alte Bräuche zurückgreifen, die unter den damaligen Baumeistern verbreitet waren und von ihnen Generation um Generation weitergegeben wurden, bis hin zu den großen Bauten der Eisenzeit. Auch im entfernten Dahistan.

Wie die erste französisch-turkmenische Ausgrabung in Geoktchik Depe zeigte, schien der monumentale Gebäudekomplex die Summe zweier großer Bauten: die erste, nördliche, barg in ihrer Mitte einen großen, viereckigen Raum (Nördliches Gebäude: 16,20 x 15,20 m, etwa 250 m2 Oberfläche und 13,40 m Höhe). Stratigraphische Schnitte im Osten, Norden und Westen, belegten den Gebrauch von Lehmziegelverbänden in einem großen Teil der, diesen Raum umgebenden Masse. Dadurch schienen die Aussenmauern dieses Raumes breiter, als in Wirklichkeit (wenngleich sie schon ziemlich dick waren) und die größte angegebene Breite $\left(25 \mathrm{~m}\right.$ im Westen $\left.{ }^{52}\right)$ bezieht sich, meines Erachtens, mehr auf die äusseren Dimensionen, die man an der Oberfläche wahrnimmt, als auf die wirkliche Breite der Mauern. Da die Arbeiten vom CNRS aus verwaltungstechnischen Gründen unterbrochen wurden, wurde keiner der Schnitte wirklich zu Ende geführt. Und so lassen mich die unveröffentlichten Fotografien und Zeichnungen, die mir Dr. O Lecomte großzügig zusammen mit seinen persönlichen Erläuterungen, zur Verfügung gestellt hat, vermuten, dass wenngleich es stimmt, dass die Mauern des Nördlichen Gebäudes ausserordentlich dick waren und einen regelmässigen Verband aus den großen, für das archaische Dahistan typischen Lehmziegeln zeigten $(50 \times 70 \text { x 8/10 cm })^{53}$, wozu noch eine innere Verkleidung hinzukam, ein Großteil der gesamten Breite in Wirklichkeit $\mathrm{zu}$ einem riesigen, massiven Konglomerat gehörte, wie wir jetzt durch unsere eigene Grabung belegen. Das Südliche Gebäude, das auf der südlich angebauten Plattform errichtet worden war, war von Brunnen und Räumen, die aus späterer Zeit stammten, durchsetzt. Darunter wäre vor allem der sogenannte Raum R485 zu erwähnen.

\footnotetext{
${ }^{50}$ V. M. Masson.- Op. cit. (1988): 56-59 und Abb. 18.

${ }^{51}$ V. M. Masson.- Op. cit. (1988): Abb. 18.

${ }^{52}$ O. Lecomte.- Op. cit. (1999): 153

${ }^{53}$ O. Lecomte.- Op. cit. (1999): 149
} 


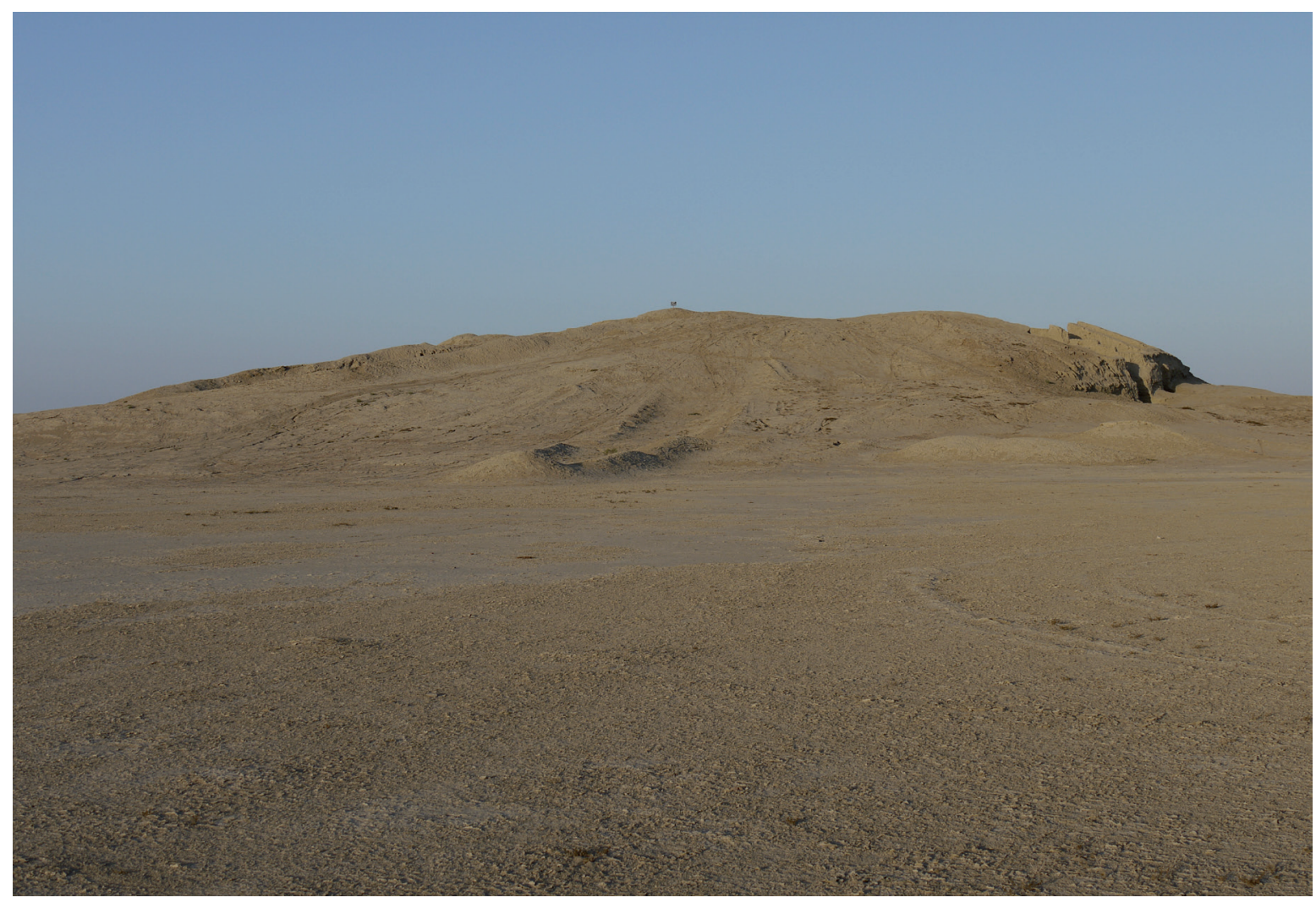

Abb. 9.- Blick auf Geoktchik vom W (Photo: Spanische und Turkmenische Mission).

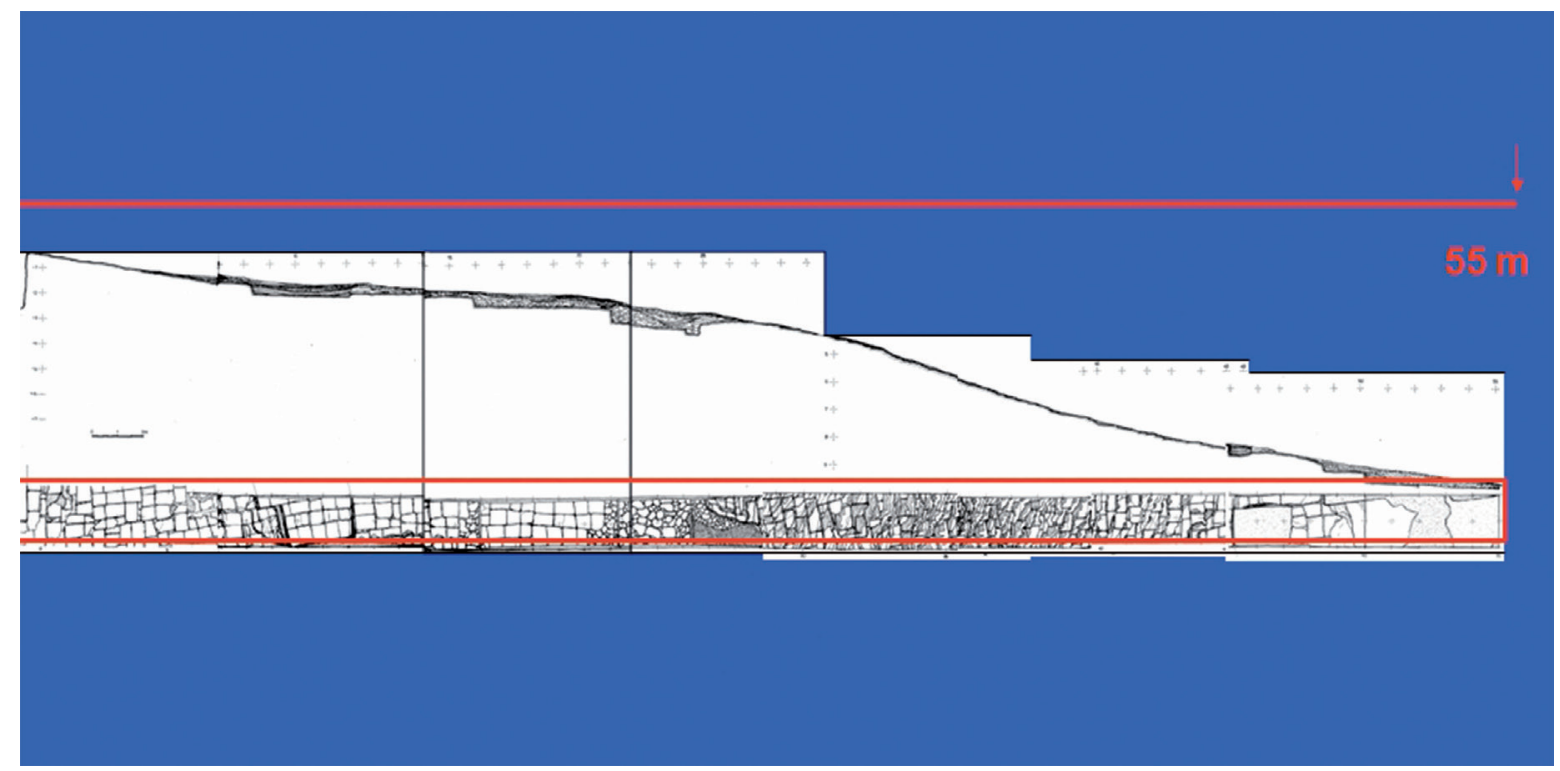

Abb. 10.- Plan und Abschnitt der südlichen Ausgrabung, vor der südlichen Erweiterung (Zeichnung: M. Á. Núnez. Versammlung: Spanische und Turkmenische Mission).

In Geoktchik (Abb. 9) versucht unser spanisch-turkmenisches Team im Moment die Form des gesamten Gebäudekomplexes, so wie seine Bautechnik, zu bestimmen. Später werden wir versuchen, die Funktion des großen Raumes im Nördlichen Gebäude herauszufinden. Dafür 
müssen wir den restlichen Sand und Schutt entfernen. ${ }^{54}$ Am Anfang der Grabung öffneten wir einen großen stratigraphischen Schnitt vom südlichen Rand der Mauer 410 (Süden) des großen Raumes im Nördlichen Gebäude bis zum Fuße des heutigen Hügels (Abb. 10). Hier konnten wir ein zweites Gebäude aus den Lehmziegeln des Typs der archaischen Dahistan-Kultur belegen, das $65 \mathrm{~m}$ lang und $12 \mathrm{~m}$ hoch war. Dieser Schnitt wurde mehrere male erweitert und entlang des westlichen Hanges des Hügels weitergeführt, mit der Absicht, so den Rand eines Viertels des Komplexes zu bestimmen. Im Moment haben wir fast ein Viertel eines Gebäudes freigelegt, das seinerzeit riesig gewesen sein muss, mit seinen 11 bis 14 m Höhe über dem ursprünglichen Boden -der tiefer liegt, als die heutige Ebene- 90m Länge und $80 \mathrm{~m}$ Breite. Die Aussenoberfläche des Gebäudes beträgt $436 \mathrm{~m} 2$. Der Verband ist äußerst regelmäßig und von guter Qualität (Abb. 11 und 12), mit den rechteckigen Lehmziegeln, die typisch für die archaische Dahistan-Kultur sind ${ }^{55}$. Unsere Maße sind die selben, wie die, die die französischturkmenische Expedition angibt. Der Verband der Gebäude ist gut gemacht und regelmäßig und wird durch Mörtel von der selben Machart wie die Lehmziegel zusammengehalten.

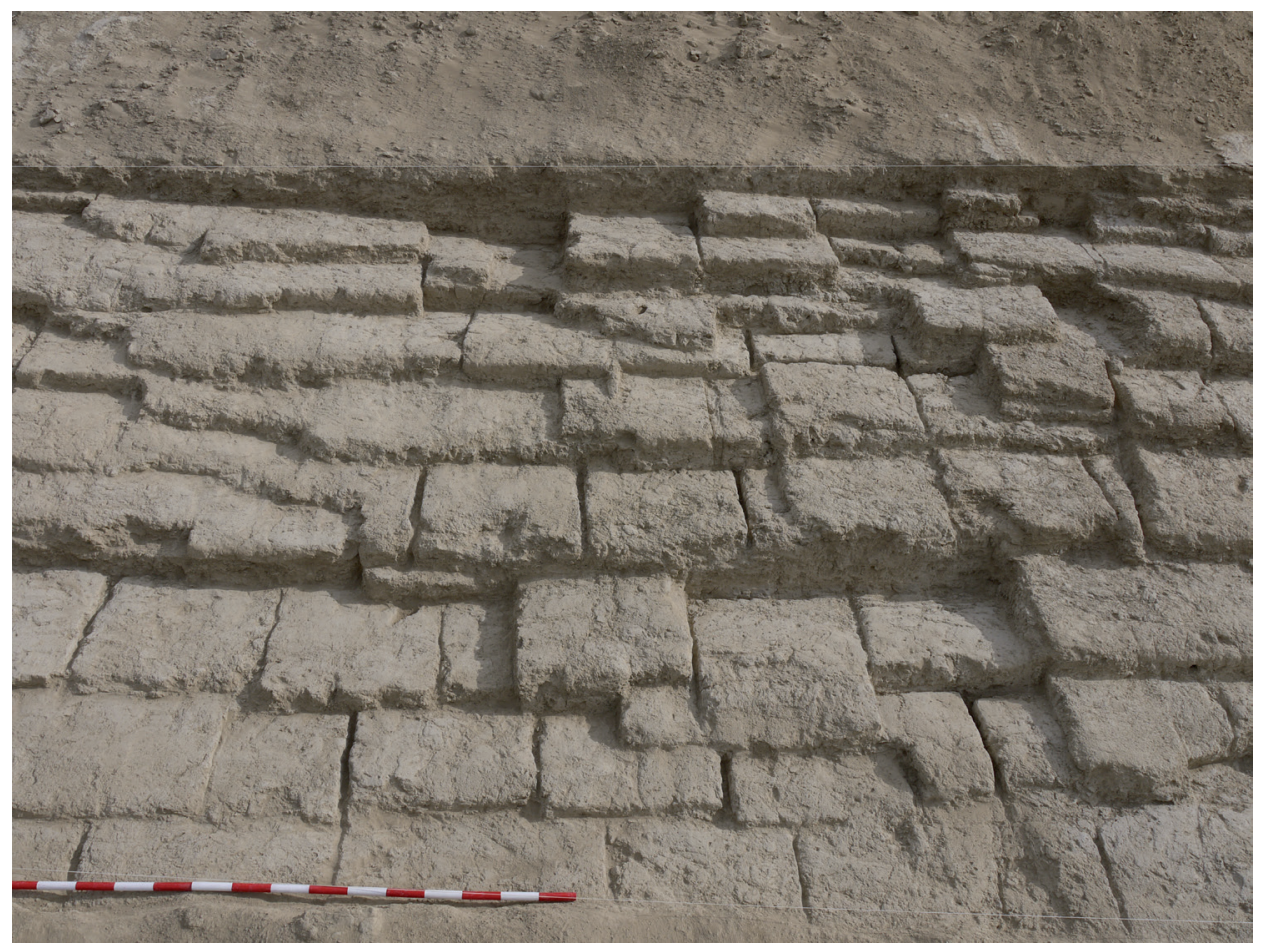

Abb. 11.- Mauerwerk auf der Südseite von Geoktchik (Photo: Spanische und Turkmenische Mission).

\footnotetext{
${ }^{54}$ Wenn man von einer kleinen Ecke absieht, muss noch eine massive Füllung von $6 \mathrm{~m}$ Erde entfernt werden, die jetzt die Lehmziegelverkleidung der inneren Wände und die fünf Räume, die sich an der nördlichen Umfassungsmauer öffnen, bedeckt. Auf dem jetzt noch verdeckten Boden, so wie in diesen fünf Räumen, sollten wir Elemente finden, die die Funktion des Komplexes erklären. Meines Erachtens würden sehr viel frühere Beispiele, wie Raum 9 im Altyn Depe des dritten Jahrtausends v. C., das in Wirklichkeit eine Art Begräbnisstätte ist (V. M. Masson, Y. E. Berioskin.- The Chronology of the Eneolithic - Middle Bronze Ages in Central Asia (Buriaals of Altyn Depe), St.Petersburg Institute of History RAS, St.-Petersburg 2005: Plate 49), mit seinen fünf kleinen Kammern in einer der Seitenwände, den Ursprung einer Tradition aufzeigen, die in der Eisenzeit den neuen Begräbnisriten angepasst worden wäre. Meiner Meinung nach praktizierte vielleicht die Oberschicht Dahistans eine Entfleischung der Körper, die dann zu diesem Zweck in diesem Raum niedergelegt worden wären.

${ }^{55}$ J. Ma Córdoba, M. Mamedov.- Op. cit. (2016): 606-609. J. Mª Córdoba.- "La cultura de la Edad del Hierro en Dehistán (1300-500 a. C.). Nuevos trabajos en Geoktchik Depe e Izat Kuli (2014-2015)", Informes y Trabajos 14 (2016): 188-207. Siehe SS. 191-194.
} 


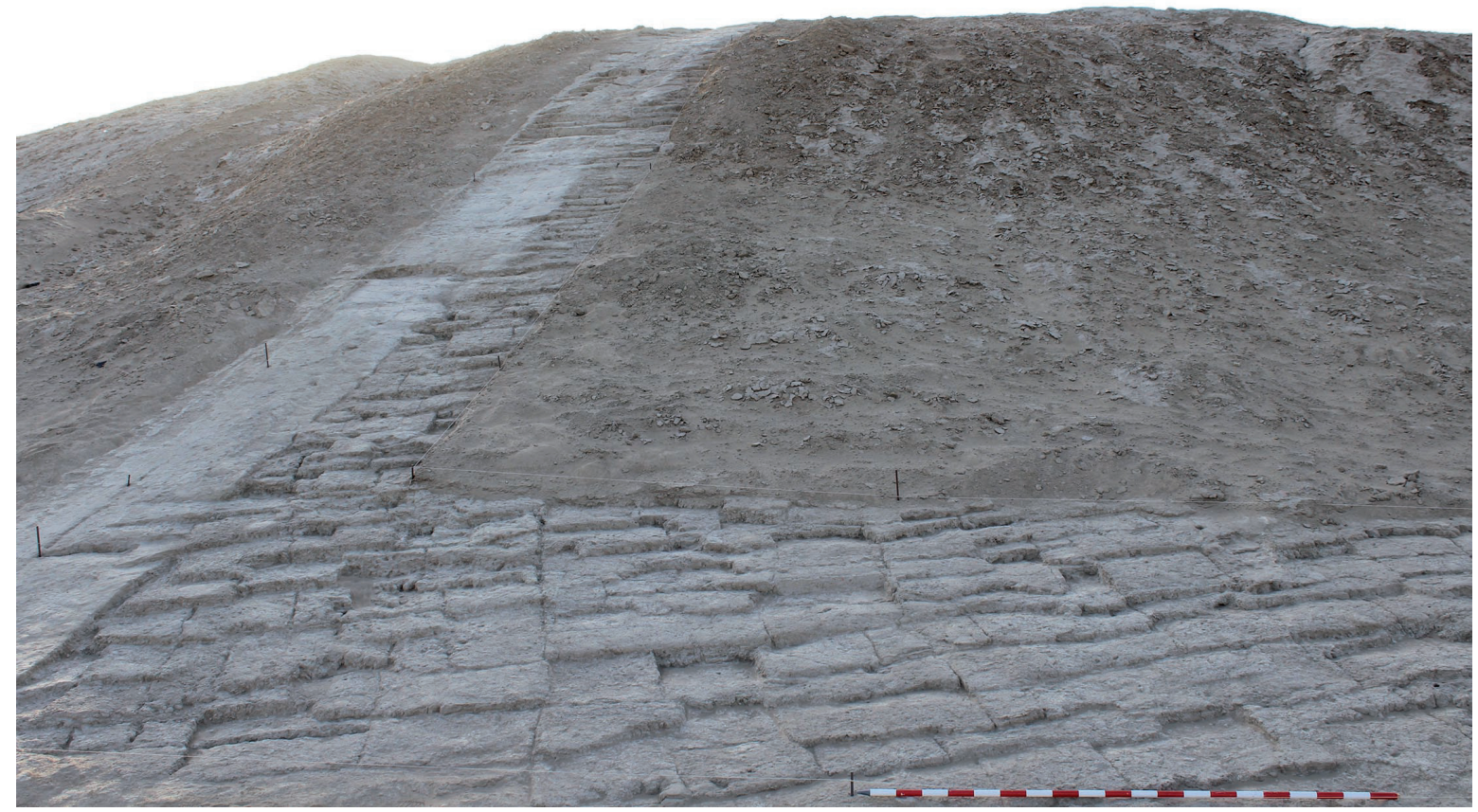

Abb. 12.- Mauerwerk auf der westlichen Seite, während der Ausgrabung (Photo: Spanische und Turkmenische Mission).

Was aber besonders hervorzuheben wäre, ist, dass wir, als wir diesen südlichen stratigraphischen Schnitt ausgruben, sahen, dass das, was ein aus Millionen Lehmziegeln errichteter, riesiger Bau schien, in Wirklichkeit ein großes Gebäude mit dicken Lehmziegelmauern war (das Nördliche Gebäude) gegen die man gewaltige Mengen gestampfter Erde und Stampflehm oder Paksha aufgehäuft hatte, die wahrscheinlich wieder durch Lehmziegelreihen gehalten wurden, obwohl wir dieses letztere noch bestätigen müssen. Den Hang, der so entstand, bedeckte man mit einem Lehmziegelverband, der in gestuften Schichten angelegt war (die wir noch nicht ganz nachzeichnen können). So wurden, ohne der Monumentalität des Ganzen Abstrich zu machen, die Baukosten verbilligt.

Dieses Gebäude zeigt uns die Techniken der Baumeister dieser Gegend, die denen ähnlich sind, die man in Takhirbai findet. Immer, wenn wir zufällig oder aus irgendeinem Grunde, an einer Stelle der, mit Lehmziegeln verkleideten, Oberfläche gruben, schienen die regelmäßigen Lehmziegelschichten sich in Schichten aus gestampfter Erde und/oder Paksha "aufzulösen". Diese Tatsache, so wie die Masse aus Paksha, die O. Lacomte auf dem oberen Teil des südlichen Gebäudes feststellte und meine eigenen Beobachtungen an den verwitterten Profilen des alten stratigraphischen Schnittes im Osten, unterstützen, meines Erachtens, unsere Hypothese. Ein tiefer, senkrechter Schnitt vom höchsten Punkt bis zum Fuße des Hügels, den wir für die nächste Grabungskampagne planen, wird -so hoffen wir- diese Frage lösen. 


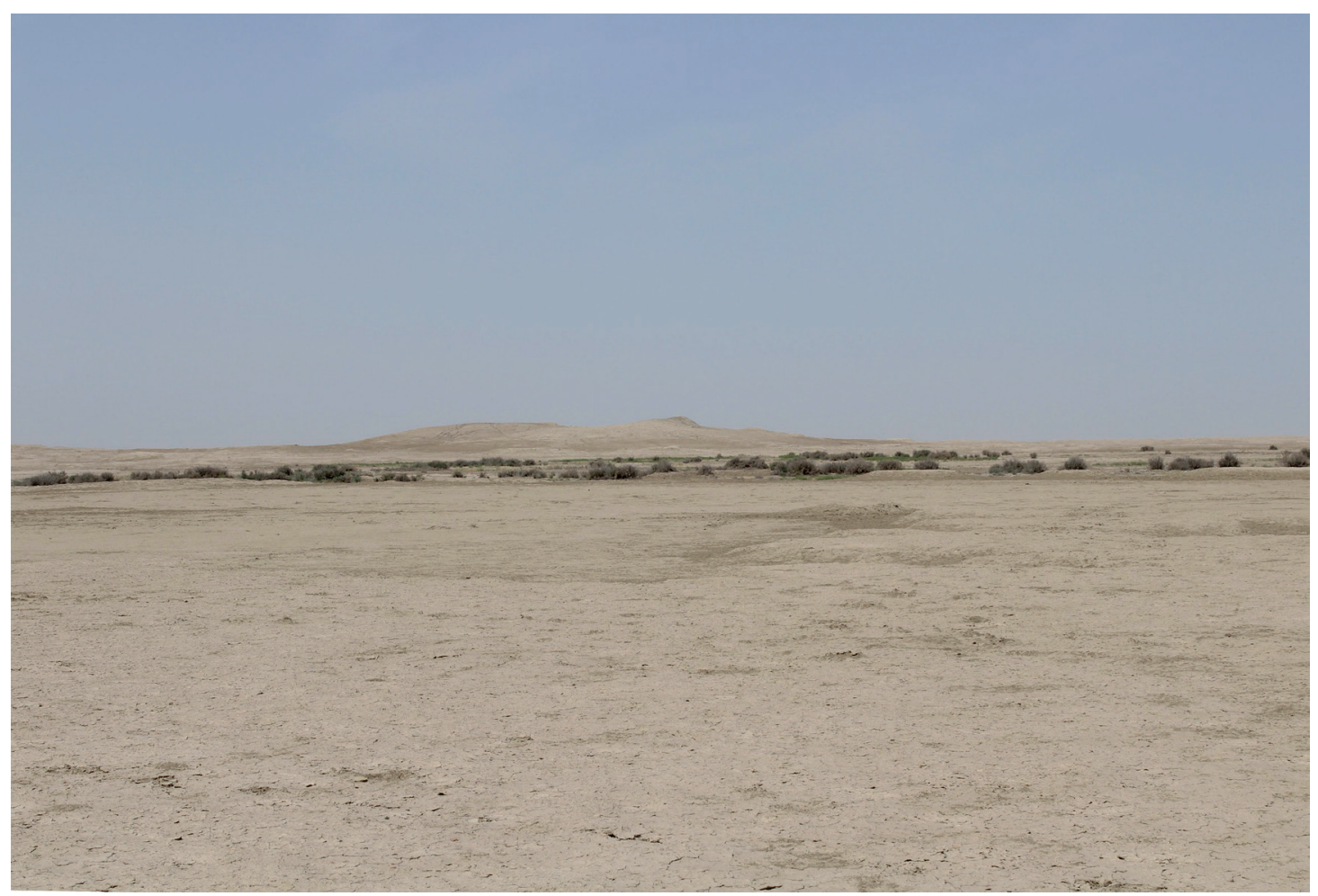

Abb. 13.- Allgemeine Ansicht des zentralen Hügels von Izat Kuli, wo sich die Plattform erhebt. Aus dem NE genommen, innerhalb der landwirtschaftlichen Fläche und Umsetzung von Nachbarschaften und Gehöfte (Foto: Spanische und Turkmenische Mission).

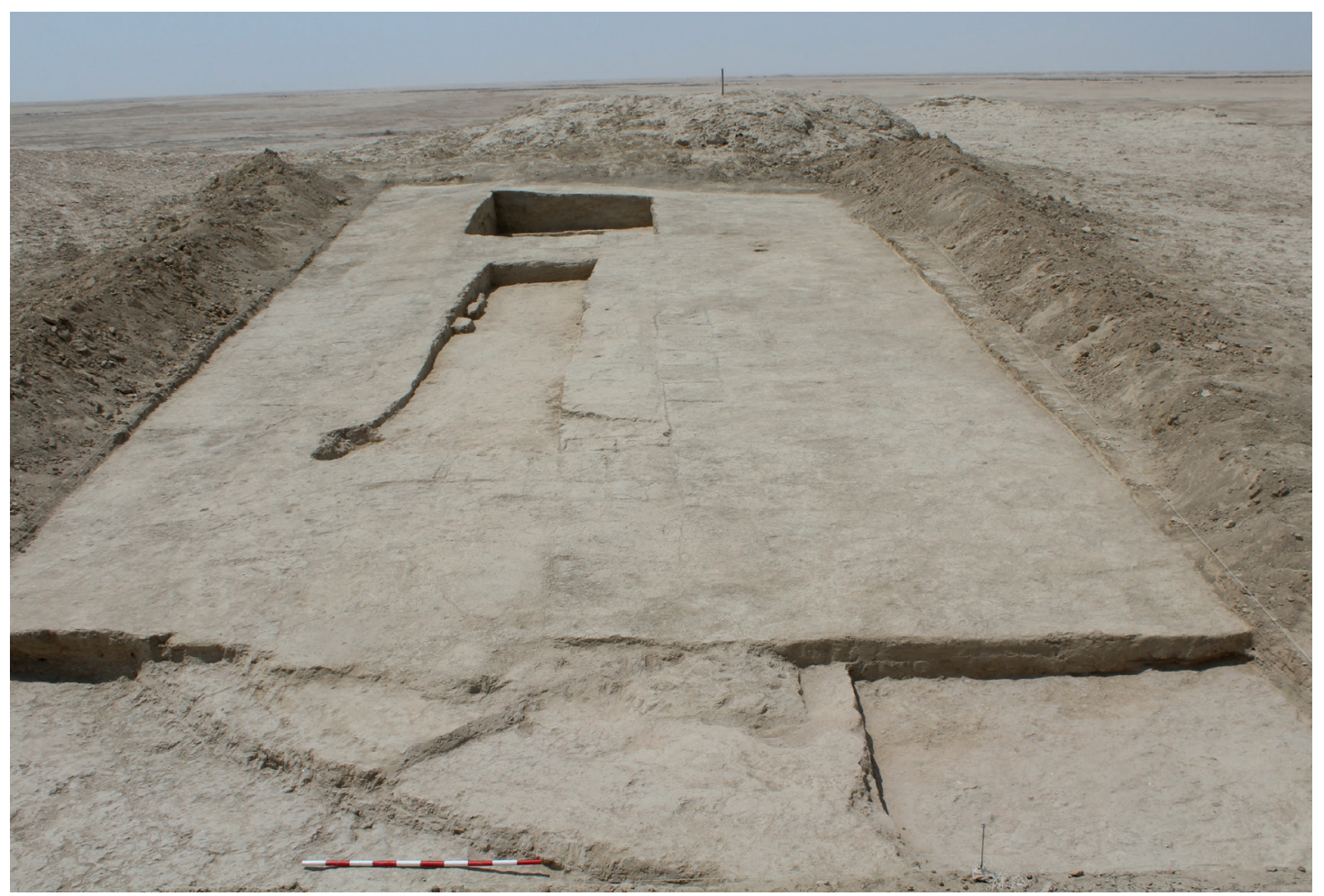

Abb. 14.- Blick auf das S eines Sektor des Fussbodens der Izat Kuli-Plattform (Photo: Spanische Mission). 
Nun ist es wahr, das Geoktchik keine normale Plattform ist, sonder ein riesiges Bauwerk. Aber was wir dort hinsichtlich der von den Baumeistern angewandten Techniken gesehen haben (Massen aus Paksha und Verkleidung aus Lehmziegelverband) wiederholt sich in der echten Plattform in Izat Kuli. In der Mitte dieser (Abb. 13), durch zwei große Kanäle abgegrenzter Grabungsstätte, deren Topographie klar eine befestigte Zitadelle und eine Plattform zeigt (die schon von V. M. Masson aufgezeigt wurden), haben wir einen Teil der Oberfläche (Abb. 14) dieser Lehmziegel-Plattform gefunden, auf der mindestens ein besonderes Gebäude stand (Gebäude 1), das wahrscheinlich eine religiöse Funktion hatte, und das wir zur Zeit ausgraben ${ }^{56}$. Ein paar Meter östlich dieses Gebäudes, haben wir einen stratigraphischen Schnitt durchgeführt. Wir wollten hier die Bauweise untersuchen und die tieferen Schichten erreichen, die älter als die Plattform waren. Anhand der tiefen Sondierung, die E. Muradova Jahre vorher $20 \mathrm{~m}$ nordöstlich unserer heutigen Grabung geöffnet hatte, in deren Stratigraphie, sich Lehmziegel- und Wohnungsschichten abwechselten ${ }^{57}$, nahm ich an, dass das monumentale Gebäude auf dem Hügel einer früheren Siedlung errichtet worden war. Bisher sind wir nur etwa $2 \mathrm{~m}$ tief gedrungen, und können dies noch nicht wissen, doch die ersten Ergebnisse unseres stratigraphischen Schnittes sind sehr erfreulich gewesen, was die Bautechniken angeht, die bei der, von den Herrschern einer sicher ausserordentlich bedeutenden Siedlung verordneten, Errichtung dieser großen Plattform, angewendet worden waren.

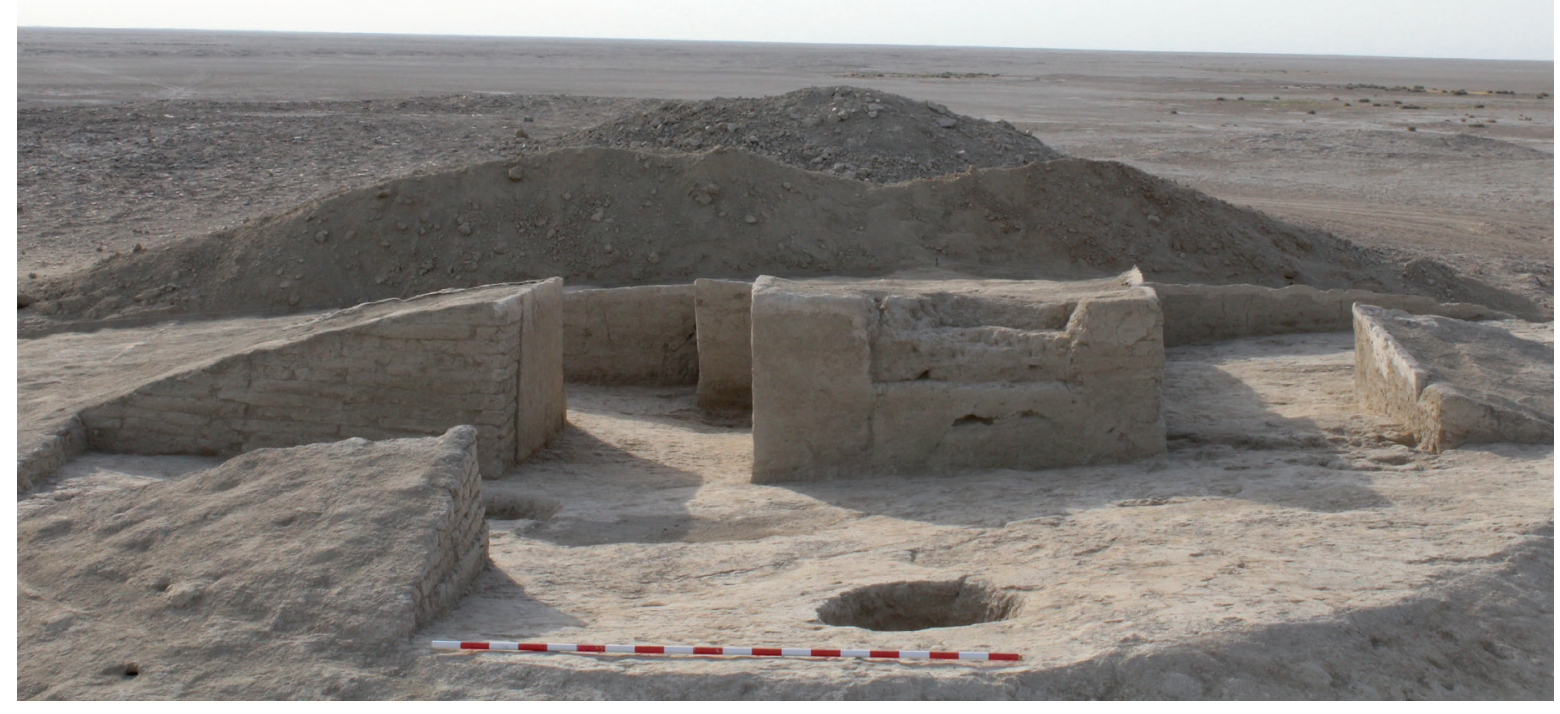

Abb. 15.- Teilansicht auf das E von Gebäude 1, aufgestellt auf der Plattform von Izat Kuli (Photo: Spanische und Turkmenische Mission).

Unser Schnitt misst 5 x 3m. Aufgrund der enormen Härte des Materials und der wenigen Zeit, die wir für unsere Ausgrabung hatten, konnten wir während der letzten Kampagne, wie schon gesagt, nur eine Tiefe von 2,30m erreichen. Aber dies hat genügt um festzustellen, dass

\footnotetext{
${ }^{56}$ J. Ma . Córdoba, M. Mamedov.- Op. cit. (2016): 609-612. J. Mª Córdoba.- Op, cit. (2016): 195-199.

${ }^{57}$ E. Muradova.- Op. cit. (1991): 52.
} 
die Plattform, auf der man besondere Gebäude (Abb. 15), wie z. B. Gebäude 1, errichtete, aus Schichten lehmiger, gestampfter Erde (Paksha) gebaut und mit Lehmziegeln, die senkrecht zur, noch nicht freigelegten, Aussenseite der Plattform gelegt worden waren, gefestigt war ${ }^{58}$. Wir können damit sagen, dass in einer mittleren Fase der archaischen Dahistan-Kultur, die Herrscher von Izat Kuli eine enorme Plattform errichten ließen, die oben einen Boden aus rechteckigen, festen Lehmziegeln hatte, deren Maße die gleichen waren, wie in Geoktchik und dass die Seitenmauern dieser Plattform wahrscheinlich eine Verkleidung aus dem selben Material besaßen. Aber das Wichtigste dieser Entdeckung ist, dass der grösste Teil der Plattform nicht aus dichten Schichten von Lehmziegeln bestand (Abb. 16), sondern aus nicht weniger dichten aus Paksha, die gepresst und gestampft worden waren und von einer Reihe von Lehmziegeln gehalten wurden, die als eine Art Bewehrung dienten und so dem Ganzen eine größere Festigkeit verliehen.

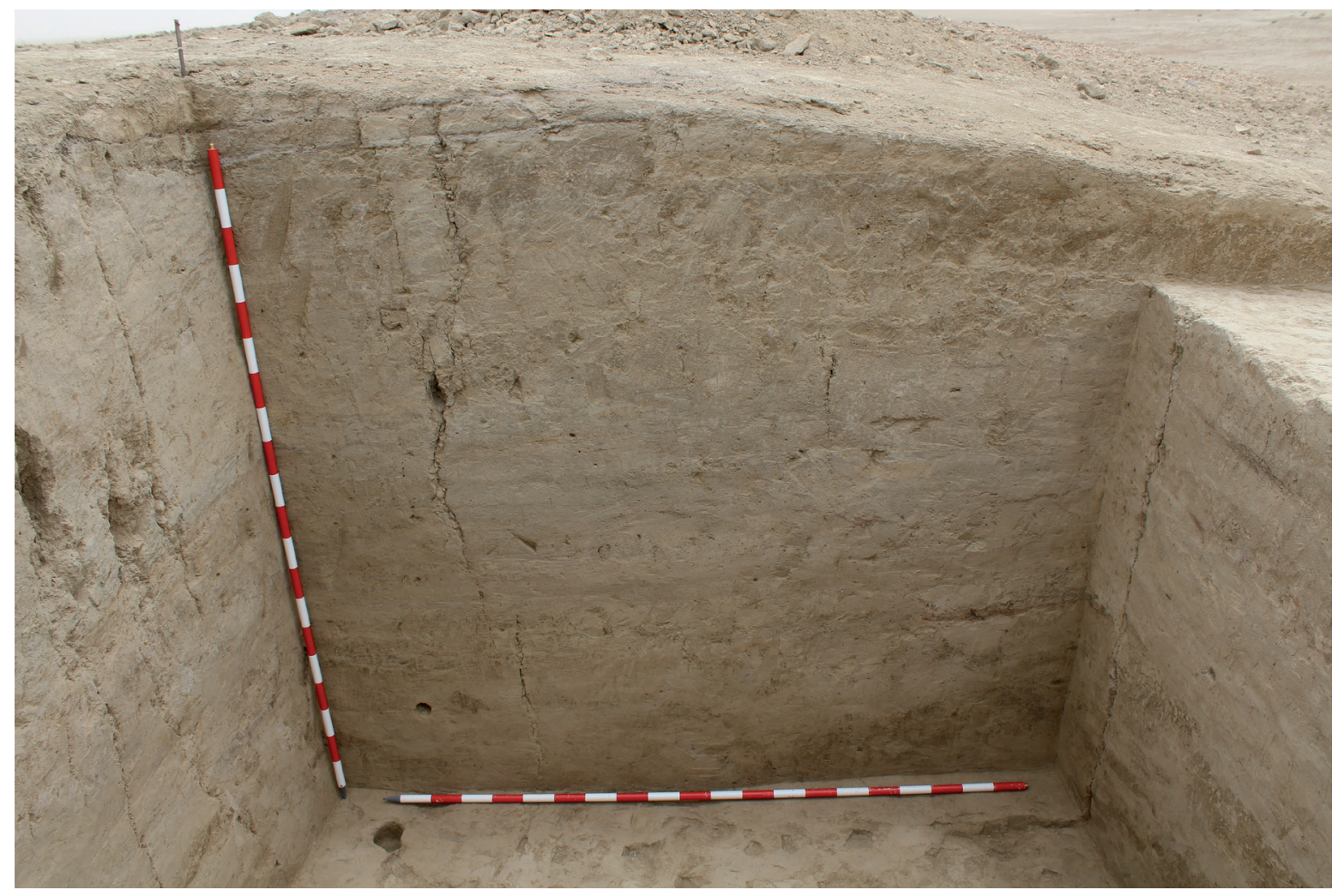

Abb. 16.- Profil E des stratigraphischen Schnitts durch die Izat Kuli-Plattform. Beachten Sie die Adobes des erhaltenen oberen Straßenpflasters, die sich in der oberen linken Ecke des Schnitts unterscheiden, sowie die starke Konsistenz der Pakhsá-Masse, die den wesentlichen Teil des Gebäudes bildet (Photo: Spanische und Turkmenische Mission).

\section{5.- Schlussfolgerungen}

Obgleich diese Schlussfolgerungen die Eindrücke einer erst an ihrem Anfang stehenden Arbeit widerspiegeln, basieren sie doch auf handfesten Ergebnissen die, meines Erachtens, gut in unsere Ausgangshypothesen passen. Es bestätigt sich eine regionale Einzigartigkeit, der von den Baumeistern angewendeten Techniken (althergebrachte Überlieferungen) und anderseits verbinden die neuen, monumentalen Bauten diese Überlieferung mit neuartigen Elementen, die von Norden und Südwesten her eingeführt worden waren. So war die große

${ }^{58}$ J. Ma Córdoba.- Op. cit. (2016): 199-200. 
Terrasse in Izat Kuli mit Techniken gebaut worden, die der alten Tradition entsprachen, wie Lehmziegel und Paksha. Sogar die Tatsache, dass sie höchstwahrscheinlich auf einem Hügel erbaut wurde, der aus Überresten älterer Siedlungen bestand und den sie dann einverleibte, kann man auf die Plattform in Altyn Depe zurückführen. Aber ihre Funktion als Unterbau der wichtigsten Gebäude der Stadt, seien sie religiös (wahrscheinlich unser Gebäude 1) oder politisch, scheint mir ein Gedanke aus den großen Städten Assyriens zu sein, der ohne Zweifel die Herrscher der neuen Staaten der Eisenzeit beeindruckt hatte.

Etwas anders liegen die Dinge in Geoktchik, dessen Plattform zwar keine solche im eigentliche Sinne ist, aber doch mit den selben Techniken errichtet wurde. Dieser Bau war wahrscheinlich eine zeremonielle Einrichtung, die vielleicht mit den Begräbnisriten der Dahistaner Oberschicht in Verbindung stand, aber klar ist, dass die Baumeister Schichten von gestampfter Erde und Paksha gegen die Wände jener riesigen Kammer auftürmten und dass der Hang, der dabei entstand, mit Lehmziegeln verkleidet wurde. Wenngleich wir noch nicht sagen können, ob dieser Bau am Ende terrassenförmig war oder nicht, gleicht die Technik jener der Tumuli oder Kurgane der nördlichen Ebenen von Andronovo. Diese Technik wäre also hier übernommen und an die Bräuche dieses Gebietes angepasst worden, sowohl an die hier übliche Architektur, wie auch an die neuen religiösen Gewohnheiten, die wahrscheinlich mit der, sich damals verbreitenden, Lehre Zarathustras verbunden waren. Und so zeigen uns in diesen Gebäuden die Baumeister des alten Vehrkānā und ihre Herrscher, gleichzeitig ihre eigene Tradition und die neuen Horizonte, die sich ihnen eröffnet hatten.

\section{BIBLIOGRAPHIE}

ATAGARRIEV, E., LISITSYNA, G. N.

1970 "Raboty nad sostablenien arkheologicheskoy karty Mesehd-Misrianskoy ravninychatskogo masiva”, Karamkumskie Drevnosti III: 166-183.

AURENCHE, O.

1977 Dictionnaire illustré multilingüe de l'architecture du Proche Orient ancient. Maison de l'Orient, Lyon.

BLESA CUENCA, J. L.

2018 Los arios. Historia y modos de vida de los pueblos centroasiáticos de la Edad del Hierro. Universidad Autónoma de Madrid, Madrid, (tesis doctoral no publicada).

CATTANI, M.

1998 "Excavations at Takhirbaj tepe (THR-1) (1992-1993). Preliminary notes", en A. Gubaev, G. A. Koshelenko, M. Tossi (dir.).- The Archaeologicl Map of the Murghab delta. Preliminary Reports 1990-95. Istituto Italiano per l'A frica e l'Oriente, Roma: 97-104.

CÓRDOBA, J. M"

2015-2016 “"De re rustica» ... in extrema pars mundi. Agricultura, parcelas y canales en Dehistán durante la Edad del Hierro (1500-500 a. C.). Notas en Izat Kuli”, Isimu 18-19: 391-406.

2016 "La cultura de la Edad del Hierro en Dehistan (1300-500 a. C.). Nuevos trabajos en Geoktchik Depe e Izat Kuli (2014-2015)”, Informes y trabajos 14: 188-207. 
CÓRDOBA, J. Ma., MAMEDOV, M.

2016 "L'âge du fer au Dehistan. Nouvelles recherches archéologiques turkmènes et espagnoles sur les sites de Geoktchik Depe et Izat Kuli (Province de Balkan, Turkmenistan)”, en O. Kaelin, H.-P. Mathys (eds.).- Proceedings of the 9th International Congress on the Archaeology of the Ancient Naer East, Harrassowitz Verlag, Wiesbaden: 601-614.

DETHIER, J.

1986 Architectures de terre. Éditions du Centre Pompidou, Paris.

EBELLING, E.

1981 “Baumeister”, RLA 1: 438-439.

GELIN, M.

1996, "L'argile comme matériau de construction: le pahsa (pisé) et la brique crue", Dossiers d'Archéologie 211: 22-23.

GRAYSON, A.K.

1976 Assyrian Royal Inscriptions, I. Otto Harrassowitz, Wiesbaden.

GUTLIEV, G.

1972 "Raskopki na kholme Chialik (Chiglik-depe)" KD IV: 47-55.

JULIVERT, M.

2015 Desiertos. Clases, distribución y ocupación humana. Publicacions de la Universitat de València, València.

KHLOPIN, I.N.

1975 "Poselenie epokhi bronzi Parkhai-depe" Kratkie Soobshcheniya 142: 116-121.

KOHL, Ph.

1984 Central Asia Palaeolithic Beginnings to the Iron Age / L'Asie central des origines à l’Âge du Fer. Éditions Recherche sur las Civilisations, Paris.

KOLDEWEY, R., WETZEL, F.

1932 Die Königsburgen von Babylon. Zweiter Teil. Die Hauptburg und der Sommerpalst Nebukadnezars im Hügel Babil. J. C. Hinrichs’sche Buchhandlung, Leipzig.

KUBBA, S. A. A.

1987 Mesopotamian Architecture and Town Planning from the Mesolithic to the end of the Proto-historic Period c. 10.000-3.500 B.C. BAR International Series 367, Oxford.

KUZMINA, E. E.

2007 The Origin of the Indo-Arians. E. J. Brill, Leiden, Boston.

LACKENBACHER, S.

1982 Le roi bâtisseur. Les récits de construction assyriens des origines a Teglatphalasar III. Éditions Recherche sur les Civilisations, Paris. 


\section{LADIRAY, D.}

2002 "Les données archéologiques", en J. Perrot (dir.).- Le palais de Darius à Suse. Une résidence royale sur la route de Persépolis à Babylone. Presses de l'université ParisSorbonne, Paris: 160-223.

LECOMTE, O.

1999 "Vehrkānā y Dehistan: late farming-communities of south-west Turkmenistan from the Iron Age to the Islamic period", Parthica 1: 135-170.

LECOMTE, O., FRANCFORT, H.-P., BOUCHARLAT, R., MAMEDOW, M.

2002 "Recherches archéologiques récentes à Ulug Dépé (Turkmenistan)" Paléorient 28, nº 2: 123-132.

LOUD, G. Loud, ALTMAN, Ch. B.

1938 Khorsabad, Part I. The Citadel and the Town. The University of Chicago Press, Chicago.

MALLOWAN, M. E. L.

1966 Nimrud and its Remains. Collins, London.

MARUSHENKO, A. A.

1959 “El'ken Depe (Otchët o raskopkakh 1953, 1954 I 1956 gg.)", Trudy Instituta Istorii Arkheologii I Etnografii Akademii Nauk Kirgizskoy SSR V: 54-109.

MASSON, V. M.

1956 "Pamjatniki kultury arkhaicheskogo Dakhistana v jugo-zapadnoj Turkmenii", TRYDY JUTAKE VII: 385-458.

1959 Drevnezemledel cheskaka cultura Margianii, Nauka, Moskva.

1988 Altyn-Depe. The University Museum, University of Pennsylvania, Philadelphia.

MASSON, V. M., BERIOSKIN, Y. E.

2005 The Chronology of the Eneolithic - Middle Bronze Ages in Central Asia (Buriaals of Altyn Depe), St.-Petersburg Institute of History RAS, St.-Petersburg.

MASSON, V. M., SARIANIDI, V. I.

1972 Central Asia. Turkmenia before the Achaemenids. Thames and Hudson Southampton.

MURADOVA, E.

1991 Poseleniya arkhaicheskovo Dakhistana. Akademiya Nauk Turmenskoi CCP, Ashgabat.

NAUMANN, R.

1971 Architectur Kleinasiens von ihren Anfängen bis zum Ende der hethitischen Zeit. Verlag Ernst Wasmuth, Tübingen. 
POUGATCHENKOVA, G. A.

1996 "L'argile matériau fondamental de l'architecture bactrienne", Dossiers d'Archéologie 211: 8-21.

SAFAR, F., MUSTAFA, M. A., LLOYD, S.

1981 Eridu. Ministry of Culture and Information, Baghdad.

SANMARTÍN, J.

1999 Códigos legales de tradición babilónica. Editorial Trotta, S. A., Madrid.

SAUVAGE, M.

1998 La brique et sa mise en oeuvre en Mésopotamie. Des origins à l'époche acheéménide. Éditions Recherche sur les Civilisations, Paris.

SCHMIDT, E. F.

1953 Persepolis I. Strutures. Reliefs. Inscriptions. The University of Chicago Press, Chicago.

STROMMENGER, E.

1980 Habuba Kabira. Eine Stadt vor 5000 Jahren. Verlag Philipp von Zabern, Mainz am Rhein.

VALLAT, F.

1987 "Expéditions orientales des rois assyriens", Dossiers Histoire et Archéologie 122: 60-62.

VAN DRIEL, C., VAN DRIEL-MURRAY, C.

1983 "Jebel Aruda, the 1982 season of excavation, interim report", Akkadica 33: 1-26.

YOUKHANA, D. G.

1997 Tell es-Sawwan. The Architecture of the Sixth Millennium B.C. NABU Publicactions, London. 